\title{
Fifth-Order Mapped Semi-Lagrangian Weighted Essentially Nonoscillatory Methods Near Certain Smooth Extrema
}

\author{
Lang Wu, Dazhi Zhang, Boying Wu, and Xiong Meng \\ Department of Mathematics, Harbin Institute of Technology, Harbin 150001, China \\ Correspondence should be addressed to Boying Wu; mathwby@hit.edu.cn
}

Received 12 April 2014; Revised 26 June 2014; Accepted 26 June 2014; Published 15 July 2014

Academic Editor: Filomena Cianciaruso

Copyright (C) 2014 Lang Wu et al. This is an open access article distributed under the Creative Commons Attribution License, which permits unrestricted use, distribution, and reproduction in any medium, provided the original work is properly cited.

\begin{abstract}
Fifth-order mapped semi-Lagrangian weighted essentially nonoscillatory (WENO) methods at certain smooth extrema are developed in this study. The schemes contain the mapped semi-Lagrangian finite volume (M-SL-FV) WENO 5 method and the mapped compact semi-Lagrangian finite difference (M-C-SL-FD) WENO 5 method. The weights in the more common scheme lose accuracy at certain smooth extrema. We introduce mapped weighting to handle the problem. In general, a cell average is applied to construct the M-SL-FV WENO 5 reconstruction, and the M-C-SL-FD WENO 5 interpolation scheme is proposed based on an interpolation approach. An accuracy test and numerical examples are used to demonstrate that the two schemes reduce the loss of accuracy and improve the ability to capture discontinuities.
\end{abstract}

\section{Introduction}

The hyperbolic conservation laws are calculated in onedimensional form defined by

$$
\begin{gathered}
u_{t}+f(u)_{x}=0, \\
u(x, 0)=u_{0}(x),
\end{gathered}
$$

where $u$ and $f(u)$ can be either scalars or vectors. The fifth-order mapped semi-Lagrangian weighted essentially nonoscillatory (WENO) methods have been produced for the one-dimensional scalar case and for systems near certain smooth extrema in this study. The scheme is divided into two parts: a mapped semi-Lagrangian finite volume (M-SL-FV) WENO method and a mapped compact semi-Lagrangian finite difference (M-C-SL-FD) WENO method.

In [1], Henrick et al. proposed the mapped weighting near critical points. They contrasted the WENO 5 scheme and the mapped WENO 5 scheme and found that the WENO 5 method reached third-order accuracy at critical points, while the mapped weighted WENO 5 scheme achieved fifthorder accuracy. So the mapped WENO scheme reduced the loss of the accuracy and improved ability to capture discontinuities. Based on the theory of the mapped WENO scheme, Feng et al. used a piecewise polynomial function to propose the new mapped weights. The scheme reduced the influence of the discontinuities in the nonsmooth stencils, such that the underlying loss of accuracy was overcome [2]. In [3], Bryson and Levy designed a new method to solve the Hamilton-Jacobi equations using a mixed model consisting of mapped weights and a Godunov-type central method. This scheme reached high-order accuracy. Borges proposed a set of smoothness indicators and mapped weights for hyperbolic conservation laws. This scheme had less dissipation and achieved high accuracy, and they applied the method to twodimensional problems and found that it performed well [4].

In 1990, Lele proposed a series of compact methods, which were associated with a spectral-like solution [5]. They presented basic programmes for approximating the derivatives: a cell-centered compact scheme and midpoint interpolation. In [6], a high-order nonlinear scheme was proposed which considered a flux splitting and boundary scheme, followed by analysis of the asymptotic stability of weighted compact nonlinear scheme. Based on the WENO scheme, finite difference weighted compact programmes were developed, which associated the approximation of derivatives with the WENO scheme. In this way the method preserved the characteristics of normal compact methods and retained the ability to capture shock waves and discontinuities 
[7]. An efficient conservative compounded compact WENO scheme was proposed for shock-turbulence interaction, analyzing the resolution properties, Fourier harmonies, and boundary closures. The boundary conditions affected the stability characteristics of the scheme, so explicit boundary conditions were proposed to deal with stability [8]. Zhang et al. developed a nonlinear weighted compact method that increased higher-order precision. They used a cell-centered compact approach, a splitting technique, and characteristic projection to increase the precision. The nonlinear compact WENO method was capable of capturing discontinuities without oscillation [9].

The hybrid model of the Lagrangian and Eulerian approaches produced the semi-Lagrangian approach, inheriting the advantages of the two approaches. This approach achieved high accuracy and allowed a weaker CFL condition. The Lagrangian-Eulerian method proposed in 1974 solved a wide variety of time-dependent multidimensional fluid problems. The methodology was stable and accurate [10]. A nonoscillatory Eulerian scheme was proposed to compute two-dimensional Euler equations, level set equations, and equations of state. The level set function was used to hunt the interface in the scheme, so that the method was easily extendable to multidimensional and multilevel time integration [11]. A Lagrangian method with high-order ENO reconstruction was designed by Cheng and Shu for compressible Euler equations in which they compared figures of the four typical numerical fluxes in the Lagrangian scheme: Godunov flux, Dukowicz flux, Lax-Friedrichs flux, and Harten-Lax-van Leer contact wave flux [12]. In [13], Liu et al. combined the Lagrangian scheme with the Lax-Wendroff method to build a new scheme which was used to calculate the compressible Euler equations. The hybrid scheme was capable of saving computational cost.

In past years the semi-Lagrangian method has been very popular in transport planning [14-16]. Oscillation-free advection of interfaces, forward-trajectory global scheme, and conservative and nonconservative forms were developed for transport schemes. Crouseilles applied the semiLagrangian method to Vlasov equations. This approach easily achieved high-order time accuracy and positivity $[17,18]$. In [19-22], Qiu and Shu developed a series of semi-Lagrangian methods which allowed weaker CFL conditions and had stability, accuracy, and positivity properties.

In the present study, a mapped semi-Lagrangian WENO method is proposed for calculating hyperbolic conservation laws near certain smooth extrema. The method contains two schemes: the M-SL-FV WENO method and M-C-SLFD WENO method. The WENO scheme is widely used for hyperbolic conservation laws [23-26], but the WENO 5 scheme can only achieve third-order accuracy at certain smooth extrema. Mapped weighting has been designed to achieve an ideal order of accuracy at certain smooth extrema $[1,2]$. The semi-Lagrangian finite volume WENO scheme uses cell averages to construct the WENO scheme [23, 24], and an interpolation approach is applied to build a semiLagrangian finite difference WENO scheme $[9,27]$. In this study, the 3-total-variation-diminishing (TVD) Runge-Kutta (RK) scheme is applied to follow the backward characteristic line in a single time step for the case of variable characteristics $[23,28,29]$. As a result, the proposed schemes achieve fifthorder accuracy and overcome the potential loss of accuracy and also capture shock well.

The paper is organized as follows. The mapped finite volume WENO scheme for scalar hyperbolic conservation laws is reviewed at certain smooth extrema in Section 2. The M-SL-FV fifth-order WENO reconstruction scheme is given in Section 3. In Section 4, the compact semi-Lagrangian finite difference scheme for one-dimensional hyperbolic conservation laws is analyzed and the fifth-order mapped weighting interpolation method is presented. In Section 5, an accuracy test and numerical tests are presented regarding fifthorder M-SL-FV and M-C-SL-FD WENO methods for onedimensional hyperbolic conservation laws. The equations have certain smooth extrema, and the numerical results show that the proposed method works well in all cases. Concluding remarks and a perspective for future work are presented in Section 6.

\section{A Review of Mapped Fifth-Order Finite Volume WENO Scheme}

The mapped fifth-order WENO scheme is shown as the solution of one-dimensional scalar conservation laws, which are summarized by

$$
\begin{aligned}
& u_{t}+f(u)_{x}=0, \\
& u(x, 0)=u_{0}(x)
\end{aligned}
$$

Putting the calculation region as $[a, b]$ and adopting the following spatial discretization:

$$
a=x_{1 / 2}<x_{3 / 2}<\cdots<x_{N+(1 / 2)}=b,
$$

where $I_{i}=\left[x_{i-(1 / 2)}, x_{i+(1 / 2)}\right], i=1,2, \ldots, N$, we choose the center of the cell and cell length as $x_{i}=(1 / 2)\left(x_{i-(1 / 2)}+\right.$ $\left.x_{i+(1 / 2)}\right)$, and $\Delta x_{i}=x_{i+(1 / 2)}-x_{i-(1 / 2)}$.

For fifth-order WENO reconstruction, we first need to identify three third-order numerical fluxes. We set

$$
\begin{aligned}
& u_{i+(1 / 2)}^{(0)}=\frac{1}{3} \bar{u}_{i-2}-\frac{7}{6} \bar{u}_{i-1}+\frac{11}{6} \bar{u}_{i}, \\
& u_{i+(1 / 2)}^{(1)}=-\frac{1}{6} \bar{u}_{i-1}+\frac{5}{6} \bar{u}_{i}+\frac{1}{3} \bar{u}_{i+1}, \\
& u_{i+(1 / 2)}^{(2)}=\frac{1}{3} \bar{u}_{i}+\frac{5}{6} \bar{u}_{i+1}-\frac{1}{6} \bar{u}_{i+2} .
\end{aligned}
$$

The fifth-order linear scheme

$$
u_{i+(1 / 2)}=\frac{1}{30} \bar{u}_{i-2}-\frac{13}{60} \bar{u}_{i-1}+\frac{47}{60} \bar{u}_{i}+\frac{9}{20} \bar{u}_{i+1}-\frac{1}{20} \bar{u}_{i+2}
$$

is based on three third-order numerical fluxes $u_{i+(1 / 2)}^{(0)}, u_{i+(1 / 2)}^{(1)}$, and $u_{i+(1 / 2)}^{(2)}$, respectively:

$$
u_{i+(1 / 2)}=d_{0} u_{i+(1 / 2)}^{(0)}+d_{1} u_{i+(1 / 2)}^{(1)}+d_{2} u_{i+(1 / 2)}^{(2)} .
$$


The combination coefficients $d_{0}, d_{1}, d_{2}$ are termed linear weights.

It is noted that if the function $u(x)$ is globally smooth, the linear weights $d_{0}, d_{1}, d_{2}$ are applied to obtain highorder accuracy. However, if the scheme oscillates near discontinuities, we need the assistance of the nonlinear weights $\omega_{r}$ proposed by Liu et al. to achieve high-order accuracy in smooth regions and capture the oscillations near discontinuities [24]. The nonlinear weights $\omega_{r}$ depend on the smoothness indicators $\beta_{r}$ which evaluate the smoothness of the functions $u_{i+(1 / 2)}^{(0)}, u_{i+(1 / 2)}^{(1)}, u_{i+(1 / 2)}^{(2)}$ and is given by

$$
\beta_{r}=\sum_{l=1}^{2} \int_{I_{i}} \Delta x_{i}^{2 l-1}\left(\frac{\partial^{l} u^{(r)}(x)}{\partial^{l} x}\right)^{2} d x, \quad r=0,1,2 .
$$

These are

$$
\begin{aligned}
& \beta_{0}=\frac{13}{12}\left(\bar{u}_{i+1}-2 \bar{u}_{i+2}+\bar{u}_{i+3}\right)^{2}+\frac{1}{4}\left(3 \bar{u}_{i+1}-4 \bar{u}_{i+2}+\bar{u}_{i+3}\right)^{2}, \\
& \beta_{1}=\frac{13}{12}\left(\bar{u}_{i}-2 \bar{u}_{i+1}+\bar{u}_{i+2}\right)^{2}+\frac{1}{4}\left(\bar{u}_{i}-\bar{u}_{i+2}\right)^{2} \\
& \beta_{2}=\frac{13}{12}\left(\bar{u}_{i-1}-2 \bar{u}_{i}+\bar{u}_{i+1}\right)^{2}+\frac{1}{4}\left(\bar{u}_{i-1}-4 \bar{u}_{i}+3 \bar{u}_{i+1}\right)^{2} .
\end{aligned}
$$

The nonlinear weights are then determined by

$$
\omega_{r}=\frac{\alpha_{r}}{\sum_{s=0}^{2} \alpha_{s}}, \quad \alpha_{r}=\frac{d_{r}}{\left(\epsilon+\beta_{r}\right)^{2}}, \quad r=0,1,2,
$$

where $d_{r}$ are the linear weights and $\epsilon$ is a small and positive number to prevent the denominator becoming zero. In most of the numerical tests, $\varepsilon=10^{-6}$.

To avoid the nonlinear weights producing any loss in precision at certain smooth extrema, Henrick et al. proposed the mapped weights [1] for conservation laws, which gave perfect precision of the WENO method at certain smooth extrema. The mapped weighted $m_{r}(\omega)$ is defined as

$$
m_{r}\left(\omega_{r}\right)=\frac{\omega_{r}\left(d_{r}+\left(d_{r}\right)^{2}-3 d_{r} \omega_{r}+\omega_{r}^{2}\right)}{\left(d_{r}\right)^{2}+\left(1-2 d_{r}\right) \omega_{r}},
$$

where $\omega_{r} \in(0,1)$ and $r=0,1,2$. The function has the following properties:

$$
\begin{gathered}
m_{r}(0)=0, \quad m_{r}(1)=1, \quad m_{r}\left(d_{r}\right)=d_{r}, \\
m_{r}^{\prime}\left(d_{r}\right)=m_{r}^{\prime \prime}\left(d_{r}\right)=0 .
\end{gathered}
$$

The mapped nonlinear weights $\omega_{r}^{(M)}$ are then computed as

$$
\omega_{r}^{(M)}=\frac{\alpha_{r}^{*}}{\sum_{s=0}^{2} \alpha_{s}^{*}}, \quad \alpha_{r}^{*}=m_{r}\left(\omega_{r}\right), \quad r=0,1,2 .
$$

In our numerical tests $\varepsilon$ was taken as $10^{-40}$. The mappedweights method created high-order precision and reduced the loss of the accuracy at certain smooth extrema.
The mapped WENO of the flux $u_{i+(1 / 2)}^{-}$is given by

$$
u_{i+(1 / 2)}^{-}=\sum_{r=0}^{2} \omega_{r}^{(M)} u_{r}\left(x_{i+(1 / 2)}\right) \text {. }
$$

This is a mirror symmetric about $x_{i}$ of the abovementioned process for the approximations $u_{i-(1 / 2)}^{+}$.

\section{M-SL-FV Fifth-Order WENO Reconstruction}

The semi-Lagrangian finite volume method and mapped fifth-order WENO reconstruction scheme for scalar and system of conservation laws are presented in this section. We first show the semi-Lagrangian finite volume scheme and then introduce the mapped WENO 5 reconstruction.

3.1. Semi-Lagrangian Finite Volume Scheme for Scalar Case. Firstly, we integrate (2) to obtain the finite volume scheme

$$
\frac{d}{d t} \bar{u}_{i}(t)=-\frac{1}{\Delta x_{i}}\left(\widehat{f}_{i+(1 / 2)}-\widehat{f}_{i-(1 / 2)}\right) .
$$

The proposed semi-Lagrangian finite volume scheme is based on integrating (14); that is, we set up the integral in time $\left[t^{n}, t^{n+1}\right]$ such that

$$
\bar{u}_{i}^{n+1}=\bar{u}_{i}^{n}-\frac{1}{\Delta x_{i}} \int_{t^{n}}^{t^{n+1}}\left(\widehat{f}_{i+(1 / 2)}-\widehat{f}_{i-(1 / 2)}\right) d t
$$

Here, the three-point Gaussian quadrature formula is used to approximate the integration in time, which limits the WENO reconstruction to fifth-order accuracy at most. From the above-mentioned estimate, the corresponding equation is acquired as

$$
\begin{aligned}
\bar{u}_{i}^{n+1}=\bar{u}_{i}^{n}-\frac{\Delta t}{\Delta x_{i}} \sum_{l=1}^{3} \rho_{l}(\widehat{f} & \left(u\left(x_{i+(1 / 2)}, t^{n}+\tau_{l} \Delta t\right)\right) \\
& \left.-\widehat{f}\left(u\left(x_{i-(1 / 2)}, t^{n}+\tau_{l} \Delta t\right)\right)\right),
\end{aligned}
$$

where $\rho_{1}=\rho_{3}=5 / 18, \rho_{2}=4 / 9$ are the weights, and $\tau_{1}=$ $(1 / 2)(1+\sqrt{0.6}), \tau_{2}=1 / 2, \tau_{3}=(1 / 2)(1-\sqrt{0.6})$ are the Gaussian quadrature points.

It should be noted that the $\widehat{f}\left(u\left(x_{i+(1 / 2)}, t^{n}+\tau_{l} \Delta t\right)\right), l=$ $1,2,3$ are not approximated by the WENO reconstruction directly, so we need to make use of the characteristic curves given by

$$
\begin{gathered}
x^{\prime}(t)=-f^{\prime}(u(x, t)), \\
x_{0}=x\left(t=t^{n}\right)=x_{i+(1 / 2)} .
\end{gathered}
$$


On the one hand, if $f^{\prime}(u)$ is variable, and the third-order TVD RK method is applied to track the characteristic curves, we find the point $x_{i+(1 / 2)}^{(3)}$ at time level $t^{n}$, which is built up by

$$
\begin{aligned}
& x_{i+(1 / 2)}^{(1)}=x_{0}-f^{\prime}\left(x_{0}, t^{n}\right) \tau_{l} \Delta t, \\
& x_{i+(1 / 2)}^{(2)}=\frac{3}{4} x_{0}+\frac{1}{4} x_{i+(1 / 2)}^{(1)}-\frac{1}{4} f^{\prime}\left(x_{i+(1 / 2)}^{(1)}, t^{n}+\tau_{l} \Delta t\right) \tau_{l} \Delta t, \\
& x_{i+(1 / 2)}^{(3)}=\frac{1}{3} x_{0}+\frac{2}{3} x_{i+(1 / 2)}^{(2)}-\frac{2}{3} f^{\prime}\left(x_{i+(1 / 2)}^{(2)}, t^{n}+\frac{1}{2} \tau_{l} \Delta t\right) \tau_{l} \Delta t .
\end{aligned}
$$

On the other hand, if $f^{\prime}(u)$ is constant, the point $x_{i+(1 / 2)}^{(3)}$ can be built up by the formula

$$
x_{i+(1 / 2)}^{(3)}=x_{0}-f^{\prime}\left(x_{0}, t^{n}\right) \tau_{l} \Delta t, \quad l=1,2,3 .
$$

Until now we have needed to substitute the above results of the reduction $x_{i+(1 / 2)}^{(3)}$ into (16) and evaluate the numerical flux $f$ at the cell point, so the semi-Lagrangian finite volume scheme is given by

$$
\begin{aligned}
\bar{u}_{i}^{n+1}=\bar{u}_{i}^{n}-\frac{\Delta t}{\Delta x_{i}} \sum_{l=1}^{3} \rho_{l}( & \widehat{f}\left(u\left(x_{i+(1 / 2)}^{(3)}, t^{n}\right)\right) \\
- & \left.\widehat{f}\left(u\left(x_{i-(1 / 2)}^{(3)}, t^{n}\right)\right)\right) .
\end{aligned}
$$

It is clear that the above result depends on the numerical flux $u$ at $x_{i+(1 / 2)}^{(3)}$ at time level $t^{n}$. Lastly, we need to reconstruct the point value $u$ at $x_{i+(1 / 2)}^{(3)}$ at time level $t^{n}$, which is described in detail in Section 3.3.

3.2. Semi-Lagrangian Finite Volume Scheme for System. Take the following one-dimensional system of conservation laws in this section, defined by

$$
\mathbf{u}_{t}+\mathbf{f}(\mathbf{u})_{x}=0,
$$

where

$$
\mathbf{u}=\left(\begin{array}{c}
u_{1} \\
u_{2} \\
\vdots \\
u_{m}
\end{array}\right), \quad \mathbf{f}(\mathbf{u})=\left(\begin{array}{c}
f_{1}(\mathbf{u}) \\
f_{2}(\mathbf{u}) \\
\vdots \\
f_{m}(\mathbf{u})
\end{array}\right)
$$

To construct the semi-Lagrangian finite volume scheme for system (21), a similar derivation for the scalar case is applied to (21), as

$$
\begin{aligned}
\overline{\mathbf{u}}_{i}^{n+1}=\overline{\mathbf{u}}_{i}^{n}-\frac{\Delta t}{\Delta x_{i}} \sum_{l=1}^{3} \rho_{l}( & \widehat{\mathbf{f}}\left(\mathbf{u}\left(x_{i+(1 / 2)}, t^{n}+\tau_{l} \Delta t\right)\right) \\
& \left.-\widehat{\mathbf{f}}\left(\mathbf{u}\left(x_{i-(1 / 2)}, t^{n}+\tau_{l} \Delta t\right)\right)\right),
\end{aligned}
$$

where $\rho_{1}=\rho_{3}=5 / 18, \rho_{2}=4 / 9, \tau_{1}=(1 / 2)(1+\sqrt{0.6}), \tau_{2}=1 / 2$ and $\tau_{3}=(1 / 2)(1-\sqrt{0.6})$, and $\widehat{\mathbf{f}}$ is a Lax-Friedrichs flux with

$$
\alpha=\max _{\substack{\mathbf{u} \\ j=1,2, \ldots, m}}\left|\lambda_{j}(\mathbf{u})\right| .
$$

Note that the characteristics are defined by integrating the eigenvalues of $\mathbf{f}^{\prime}(\mathbf{u})$ for the case where the initial value problem of the system is to meet the characteristic curves

$$
\begin{gathered}
x^{\prime}(t)=-\lambda_{p}(\mathbf{u}(x, t)), \quad p=1,2, \ldots, m, \\
x_{0}=x\left(t=t^{n}\right)=x_{i+(1 / 2)} .
\end{gathered}
$$

The third-order TVD RK method is used to solve the abovementioned initial value problem. The solution is obtained from

$$
\begin{aligned}
x_{i+(1 / 2)}^{(1)}= & x_{0}-\lambda_{p}\left(x_{0}, t^{n}\right) \tau_{l} \Delta t, \\
x_{i+(1 / 2)}^{(2)}= & \frac{3}{4} x_{0}+\frac{1}{4} x_{i+(1 / 2)}^{(1)} \\
& -\frac{1}{4} \lambda_{p}\left(x_{i+(1 / 2)}^{(1)}, t^{n}+\tau_{l} \Delta t\right) \tau_{l} \Delta t, \\
x_{i+(1 / 2)}^{(3)}= & \frac{1}{3} x_{0}+\frac{2}{3} x_{i+(1 / 2)}^{(2)} \\
& -\frac{2}{3} \lambda_{p}\left(x_{i+(1 / 2)}^{(2)}, t^{n}+\frac{1}{2} \tau_{l} \Delta t\right) \tau_{l} \Delta t,
\end{aligned}
$$

where $p=1,2, \ldots, m$. In a derivation similar to the scalar case, the semi-Lagrangian finite volume scheme for the system (21) is reduced to give

$$
\begin{aligned}
& \overline{\mathbf{u}}_{i}^{n+1}=\overline{\mathbf{u}}_{i}^{n}-\frac{\Delta t}{\Delta x_{i}} \sum_{l=1}^{3} \rho_{l}( \widehat{\mathbf{f}}\left(\mathbf{u}\left(x_{i+(1 / 2)}^{(3)}, t^{n}\right)\right) \\
&\left.-\widehat{\mathbf{f}}\left(\mathbf{u}\left(x_{i-(1 / 2)}^{(3)}, t^{n}\right)\right)\right) .
\end{aligned}
$$

The WENO reconstruction is provided in detail in Section 3.3.

3.3. Mapped Fifth-Order WENO Reconstruction. Ordinarily, fifth-order WENO reconstruction maintains fifth-order accuracy in smooth regions and captures discontinuities well but only achieves third-order accuracy at certain smooth extrema. Mapped nonlinear weights are used to better approximate the numerical flux at certain smooth extrema. In this subsection, we shall first consider the case when $\lambda_{p} \geq 0$; then we will consider the case when $\lambda_{p}<0$. The detail of the scheme has been omitted; only the necessary formulae are given in the following.

Case $1\left(\lambda_{p} \geq 0\right)$. In fifth-order reconstruction, we always use the small stencils $\left\{I_{i-2}, I_{i-1}, I_{i}\right\},\left\{I_{i-1}, I_{i}, I_{i+1}\right\}$, and $\left\{I_{i}, I_{i+1}, I_{i+2}\right\}$ to construct the second-order reconstruction polynomials $p_{r}(x)(r=0,1,2)$. Then we use the large stencils $\left\{I_{i-2}, I_{i-1}, I_{i}, I_{i+1}, I_{i+2}\right\}$ to construct the fourth-order 
reconstruction polynomial $q(x)$. Defining $\xi_{i}=\left(x_{i+(1 / 2)}-\right.$ $\left.x_{i+(1 / 2)}^{(3)}\right) / \Delta x_{i}$ we have the following reconstituted scheme:

$$
\begin{aligned}
& p_{0}\left(x_{i+(1 / 2)}^{(3)}\right)=\left(\frac{1}{3}-\xi_{i}+\frac{\xi_{i}^{2}}{2}\right) \bar{u}_{i-2}^{n}+\left(-\frac{7}{6}+3 \xi_{i}-\xi_{i}^{2}\right) \bar{u}_{i-1}^{n} \\
& +\left(\frac{11}{6}-2 \xi_{i}+\frac{\xi_{i}^{2}}{2}\right) \bar{u}_{i}^{n} \\
& p_{1}\left(x_{i+(1 / 2)}^{(3)}\right)=\left(-\frac{1}{6}+\frac{\xi_{i}^{2}}{2}\right) \bar{u}_{i-1}^{n}+\left(\frac{5}{6}+\xi_{i}-\xi_{i}^{2}\right) \bar{u}_{i}^{n} \\
& +\left(\frac{1}{3}-\xi_{i}+\frac{\xi_{i}^{2}}{2}\right) \bar{u}_{i+1}^{n} \\
& p_{2}\left(x_{i+(1 / 2)}^{(3)}\right)=\left(\frac{1}{3}+\xi_{i}+\frac{\xi_{i}^{2}}{2}\right) \bar{u}_{i}^{n}+\left(\frac{5}{6}-\xi_{i}-\xi_{i}^{2}\right) \bar{u}_{i+1}^{n} \\
& +\left(-\frac{1}{6}+\frac{\xi_{i}^{2}}{2}\right) \bar{u}_{i+2}^{n} \\
& q\left(x_{i+(1 / 2)}^{(3)}\right)=\left(\frac{1}{30}-\frac{\xi_{i}^{2}}{8}+\frac{\xi_{i}^{4}}{24}\right) \bar{u}_{i-2}^{n} \\
& +\left(-\frac{13}{60}-\frac{\xi_{i}}{12}+\frac{3 \xi_{i}^{2}}{4}+\frac{\xi_{i}^{3}}{6}-\frac{\xi_{i}^{4}}{6}\right) \bar{u}_{i-1}^{n} \\
& +\left(\frac{47}{60}+\frac{5 \xi_{i}}{4}-\xi_{i}^{2}-\frac{\xi_{i}^{3}}{2}+\frac{\xi_{i}^{4}}{4}\right) \bar{u}_{i}^{n} \\
& +\left(\frac{9}{20}-\frac{5 \xi_{i}}{4}+\frac{\xi_{i}^{2}}{4}+\frac{\xi_{i}^{3}}{2}-\frac{\xi_{i}^{4}}{6}\right) \bar{u}_{i+1}^{n} \\
& +\left(-\frac{1}{20}+\frac{\xi_{i}}{12}+\frac{\xi_{i}^{2}}{8}-\frac{\xi_{i}^{3}}{6}+\frac{\xi_{i}^{4}}{24}\right) \bar{u}_{i+2}^{n}
\end{aligned}
$$

and the corresponding linear weights, indicated by $d_{0}, d_{1}, d_{2}$ in this case:

$$
\begin{gathered}
d_{0}=\frac{1 / 30-\xi_{i}^{2} / 8+\xi_{i}^{4} / 24}{1 / 3-\xi_{i}+\xi_{i}^{2} / 2} \\
d_{1}=\left(-\frac{1}{30}+\frac{4 \xi_{i}}{45}+\frac{9 \xi_{i}^{2}}{80}-\frac{13 \xi_{i}^{3}}{36}+\frac{11 \xi_{i}^{4}}{144}+\frac{\xi_{i}^{5}}{8}-\frac{\xi_{i}^{6}}{24}\right) \\
\times\left(\left(\frac{1}{3}-\xi_{i}+\frac{\xi_{i}^{2}}{2}\right)\left(-\frac{1}{6}+\frac{\xi_{i}^{2}}{2}\right)\right)^{-1}, \\
d_{2}=\frac{-1 / 20+\xi_{i} / 12+\xi_{i}^{2} / 8-\xi_{i}^{3} / 6+\xi_{i}^{4} / 24}{-1 / 6+\xi_{i}^{2} / 2}
\end{gathered}
$$

The smoothness indicators take the following form:

$$
\begin{aligned}
& \beta_{0}=\frac{13}{12}\left(\bar{u}_{i-2}^{n}-2 \bar{u}_{i-1}^{n}+\bar{u}_{i}^{n}\right)^{2}+\frac{1}{4}\left(\bar{u}_{i-2}^{n}-4 \bar{u}_{i-1}^{n}+3 \bar{u}_{i}^{n}\right)^{2}, \\
& \beta_{1}=\frac{13}{12}\left(\bar{u}_{i-1}^{n}-2 \bar{u}_{i}^{n}+\bar{u}_{i+1}^{n}\right)^{2}+\frac{1}{4}\left(\bar{u}_{i-1}^{n}-\bar{u}_{i+1}^{n}\right)^{2}, \\
& \beta_{2}=\frac{13}{12}\left(\bar{u}_{i}^{n}-2 \bar{u}_{i+1}^{n}+\bar{u}_{i+2}^{n}\right)^{2}+\frac{1}{4}\left(3 \bar{u}_{i}^{n}-4 \bar{u}_{i+1}^{n}+\bar{u}_{i+2}^{n}\right)^{2} .
\end{aligned}
$$

The nonlinear weights satisfy

$$
\omega_{r}=\frac{\alpha_{r}}{\sum_{s=0}^{2} \alpha_{s}}, \quad \alpha_{r}=\frac{d_{r}}{\left(\epsilon+\beta_{r}\right)^{2}}, \quad r=0,1,2
$$

The mapped nonlinear weights $\omega_{r}^{(M)}$ are then computed from

$$
\omega_{r}^{(M)}=\frac{\alpha_{r}^{*}}{\sum_{s=0}^{2} \alpha_{s}^{*}}, \quad \alpha_{r}^{*}=m_{r}\left(\omega_{r}\right), \quad r=0,1,2 .
$$

Here $\epsilon>0$ is to avoid the denominator becoming zero; we used the value $\varepsilon=10^{-40}$ in our numerical tests. The numerical flux is reconstructed by the following fifth-order mapped WENO scheme with the semi-Lagrangian finite volume scheme

$$
\left(u_{i+(1 / 2)}^{(3)}\right)^{-}=\sum_{r=0}^{2} \omega_{r}^{(M)} p_{r}\left(x_{i+(1 / 2)}^{(3)}\right) .
$$

Case $2\left(\lambda_{p}<0\right)$. We use the the small stencils $\left\{I_{i+3}, I_{i+2}, I_{i+1}\right\}$, $\left\{I_{i+2}, I_{i+1}, I_{i}\right\}$, and $\left\{I_{i+1}, I_{i}, I_{i-1}\right\}$ to construct the second-order reconstruction polynomials $p_{r}(x)(r=0,1,2)$ and then use the large stencils $\left\{I_{i+3}, I_{i+2}, I_{i+1}, I_{i}, I_{i-1}\right\}$ to derive a fourthorder reconstruction polynomial $q(x)$. We have

$$
\begin{aligned}
p_{0}\left(x_{i+(1 / 2)}^{(3)}\right)= & \left(\frac{1}{3}+\xi_{i}+\frac{\xi_{i}^{2}}{2}\right) \bar{u}_{i+3}^{n}+\left(-\frac{7}{6}-3 \xi_{i}-\xi_{i}^{2}\right) \bar{u}_{i+2}^{n} \\
& +\left(\frac{11}{6}+2 \xi_{i}+\frac{\xi_{i}^{2}}{2}\right) \bar{u}_{i+1}^{n}, \\
p_{1}\left(x_{i+(1 / 2)}^{(3)}\right)= & \left(-\frac{1}{6}+\frac{\xi_{i}^{2}}{2}\right) \bar{u}_{i+2}^{n}+\left(\frac{5}{6}-\xi_{i}-\xi_{i}^{2}\right) \bar{u}_{i+1}^{n} \\
& +\left(\frac{1}{3}+\xi_{i}+\frac{\xi_{i}^{2}}{2}\right) \bar{u}_{i}^{n}, \\
p_{2}\left(x_{i+(1 / 2)}^{(3)}\right)= & \left(\frac{1}{3}-\xi_{i}+\frac{\xi_{i}^{2}}{2}\right) \bar{u}_{i+1}^{n}+\left(\frac{5}{6}+\xi_{i}-\xi_{i}^{2}\right) \bar{u}_{i}^{n} \\
& +\left(-\frac{1}{6}+\frac{\xi_{i}^{2}}{2}\right) \bar{u}_{i-1}^{n},
\end{aligned}
$$




$$
\begin{aligned}
q\left(x_{i+(1 / 2)}^{(3)}\right)= & \left(\frac{1}{30}-\frac{\xi_{i}^{2}}{8}+\frac{\xi_{i}^{4}}{24}\right) \bar{u}_{i+3}^{n} \\
& +\left(-\frac{13}{60}+\frac{\xi_{i}}{12}+\frac{3 \xi_{i}^{2}}{4}-\frac{\xi_{i}^{3}}{6}-\frac{\xi_{i}^{4}}{6}\right) \bar{u}_{i+2}^{n} \\
& +\left(\frac{47}{60}-\frac{5 \xi_{i}}{4}-\xi_{i}^{2}+\frac{\xi_{i}^{3}}{2}+\frac{\xi_{i}^{4}}{4}\right) \bar{u}_{i+1}^{n} \\
& +\left(\frac{9}{20}+\frac{5 \xi_{i}}{4}+\frac{\xi_{i}^{2}}{4}-\frac{\xi_{i}^{3}}{2}-\frac{\xi_{i}^{4}}{6}\right) \bar{u}_{i}^{n} \\
& +\left(-\frac{1}{20}-\frac{\xi_{i}}{12}+\frac{\xi_{i}^{2}}{8}+\frac{\xi_{i}^{3}}{6}+\frac{\xi_{i}^{4}}{24}\right) \bar{u}_{i-1}^{n},
\end{aligned}
$$

and the corresponding linear weights, which are indicated by $d_{0}, d_{1}, d_{2}$. In this case

$$
\begin{gathered}
d_{0}=\frac{1 / 30-\xi_{i}^{2} / 8+\xi_{i}^{4} / 24}{1 / 3+\xi_{i}+\xi_{i}^{2} / 2} \\
d_{1}=\left(-\frac{1}{30}-\frac{4 \xi_{i}}{45}+\frac{9 \xi_{i}^{2}}{80}+\frac{13 \xi_{i}^{3}}{36}+\frac{11 \xi_{i}^{4}}{144}-\frac{\xi_{i}^{5}}{8}-\frac{\xi_{i}^{6}}{24}\right) \\
\times\left(\left(\frac{1}{3}+\xi_{i}+\frac{\xi_{i}^{2}}{2}\right)\left(-\frac{1}{6}+\frac{\xi_{i}^{2}}{2}\right)\right)^{-1}, \\
d_{2}=\frac{-1 / 20-\xi_{i} / 12+\xi_{i}^{2} / 8+\xi_{i}^{3} / 6+\xi_{i}^{4} / 24}{-1 / 6+\xi_{i}^{2} / 2} .
\end{gathered}
$$

The smoothness indicators take the following form:

$$
\begin{aligned}
& \beta_{0}=\frac{13}{12}\left(\bar{u}_{i+1}^{n}-2 \bar{u}_{i+2}^{n}+\bar{u}_{i+3}^{n}\right)^{2}+\frac{1}{4}\left(3 \bar{u}_{i+1}^{n}-4 \bar{u}_{i+2}^{n}+\bar{u}_{i+3}^{n}\right)^{2}, \\
& \beta_{1}=\frac{13}{12}\left(\bar{u}_{i}^{n}-2 \bar{u}_{i+1}^{n}+\bar{u}_{i+2}^{n}\right)^{2}+\frac{1}{4}\left(\bar{u}_{i}^{n}-\bar{u}_{i+2}^{n}\right)^{2}, \\
& \beta_{2}=\frac{13}{12}\left(\bar{u}_{i-1}^{n}-2 \bar{u}_{i}^{n}+\bar{u}_{i+1}^{n}\right)^{2}+\frac{1}{4}\left(\bar{u}_{i-1}^{n}-4 \bar{u}_{i}^{n}+3 \bar{u}_{i+1}^{n}\right)^{2} .
\end{aligned}
$$

The corresponding numeration is applied to obtain the following reconstituted scheme, so the numerical flux is reconstructed by the following fifth-order mapped WENO scheme and semi-Lagrangian finite volume scheme:

$$
\left(u_{i+(1 / 2)}^{(3)}\right)^{-}=\sum_{r=0}^{2} \omega_{r}^{(M)} p_{r}\left(x_{i+(1 / 2)}^{(3)}\right) \text {. }
$$

\section{M-C-SL-FD WENO Interpolation}

The compact semi-Lagrangian finite difference method and mapped fifth-order WENO interpolation for scalar and system of conservation laws are given in this section. We first present the compact semi-Lagrangian finite difference scheme.
4.1. Compact Semi-Lagrangian Finite Difference Scheme for Scalar Case. From (2) with uniform grid $\Delta x=x_{i+(1 / 2)}-$ $x_{i-(1 / 2)}$, then at the grid point $x=x_{i}$, a semidiscrete finite different scheme

$$
\left(\frac{\partial u}{\partial t}\right)_{i}=-f_{i}^{\prime}
$$

On the basis of (38) we propose a compact semi-Lagrangian finite difference scheme. Firstly, we set up the integral in time

$$
u_{i}^{n+1}=u_{i}^{n}-\int_{t^{n}}^{t^{n+1}} f_{i}^{\prime} d t .
$$

The three-point Gaussian quadrature formula is then used to compute (39), which achieves fifth-order accuracy at most for the WENO reconstruction. From the above estimate, the corresponding equation is

$$
u_{i}^{n+1}=u_{i}^{n}-\Delta t \sum_{l=1}^{3} \rho_{l} f^{\prime}\left(u\left(x_{i}, t^{n}+\tau_{l} \Delta t\right)\right),
$$

where $\rho_{1}=\rho_{3}=5 / 18, \rho_{2}=4 / 9$ are the weights, and $\tau_{1}=(1 / 2)(1+\sqrt{0.6}), \tau_{2}=1 / 2, \tau_{3}=(1 / 2)(1-$ $\sqrt{0.6})$ are the Gaussian quadrature points. In this study, we applied the sixth-order accuracy compact scheme to propose the compact semi-Lagrangian finite difference scheme. The sixth-order accuracy compact scheme is given as [5]

$$
\begin{aligned}
\alpha f_{i-1}^{\prime}+f_{i}^{\prime}+\alpha f_{i+1}^{\prime}= & b \frac{f_{i+(3 / 2)}-f_{i-(3 / 2)}}{3 \Delta x} \\
& +a \frac{f_{i+(1 / 2)}-f_{i-(1 / 2)}}{\Delta x}
\end{aligned}
$$

where $\alpha=9 / 62, a=(3 / 8)(3-2 \alpha) ; b=(1 / 8)(22 \alpha-1)$; and $(1 / 107520)(75-354 \alpha) \Delta x^{6} f^{(7)}$ is the truncation error of the sixth-order tridiagonal scheme. By a corresponding derivation, the compact semi-Lagrangian finite difference scheme is given by

$$
\begin{aligned}
& u_{i}^{n+1} \\
& =u_{i}^{n} \\
& -\Delta t \sum_{l=1}^{3} \rho_{l}\left(c \left(\left(\widehat{f}\left(u\left(x_{i+(3 / 2)}, t^{n}+\tau_{l} \Delta t\right)\right)\right.\right.\right. \\
& \left.\left.-\widehat{f}\left(u\left(x_{i-(3 / 2)}, t^{n}+\tau_{l} \Delta t\right)\right)\right)(3 \Delta x)^{-1}\right) \\
& +d\left(\left(\widehat{f}\left(u\left(x_{i+(1 / 2)}, t^{n}+\tau_{l} \Delta t\right)\right)\right.\right. \\
& \left.\left.\left.\quad-\widehat{f}\left(u\left(x_{i-(1 / 2)}, t^{n}+\tau_{l} \Delta t\right)\right)\right)(\Delta x)^{-1}\right)\right) .
\end{aligned}
$$

From the above equation, we discover that (42) contains the corresponding compact coefficients $c$ and $d$, which are determined by (41).

Until now, with the help of the characteristic curves, we have found the points $x_{i+(1 / 2)}^{(3)}$ and $x_{i-(1 / 2)}^{(3)}$. These points 
are computed using identical arithmetic in the scalar case of the semi-Lagrangian finite volume scheme. Substituting the above results of the reduction $x_{i+(1 / 2)}^{(3)}$ into (42) and evaluating the numerical flux at the cell point, the compact semi-Lagrangian finite difference scheme is given by

$$
\begin{aligned}
& u_{i}^{n+1} \\
& =u_{i}^{n} \\
& -\Delta t \sum_{l=1}^{3} \rho_{l}\left(c \left(\left(\widehat{f}\left(u\left(x_{i+(3 / 2)}^{(3)}, t^{n}\right)\right)\right.\right.\right. \\
& \left.\left.-\widehat{f}\left(u\left(x_{i-(3 / 2)}^{(3)}, t^{n}\right)\right)\right)(3 \Delta x)^{-1}\right) \\
& +d\left(\left(\widehat{f}\left(u\left(x_{i+(1 / 2)}^{(3)}, t^{n}\right)\right)\right.\right. \\
& \left.\left.\left.\quad-\widehat{f}\left(u\left(x_{i-(1 / 2)}^{(3)}, t^{n}\right)\right)\right)(\Delta x)^{-1}\right)\right) .
\end{aligned}
$$

It is clear that the above result depends on the numerical flux $f$ at $x_{i+(1 / 2)}^{(3)}$, at time level $t^{n}$. Finally, we need to reconstruct the point value $f$ at $x_{i+(1 / 2)}^{(3)}$ at time level $t^{n}$. This is described in detail in Section 4.3.

4.2. Compact Semi-Lagrangian Finite Difference Scheme for System. Parallel to the system in the semi-Lagrangian finite volume scheme, the characteristics are defined by integrating the eigenvalues of $\mathbf{f}^{\prime}(\mathbf{u})$, denoted by $\lambda_{p}(u(x, t))$, $p=1,2, \ldots, m$. In the same way, the third-order TVD RK method is used to solve the characteristic curves. The solution is given by

$$
\begin{aligned}
& x_{i+(1 / 2)}^{(1)}=x_{0}-\lambda_{p}\left(x_{0}, t^{n}\right) \tau_{l} \Delta t, \\
& x_{i+(1 / 2)}^{(2)}=\frac{3}{4} x_{0}+\frac{1}{4} x_{i+(1 / 2)}^{(1)}-\frac{1}{4} \lambda_{p}\left(x_{i+(1 / 2)}^{(1)}, t^{n}+\tau_{l} \Delta t\right) \tau_{l} \Delta t, \\
& x_{i+(1 / 2)}^{(3)}=\frac{1}{3} x_{0}+\frac{2}{3} x_{i+(1 / 2)}^{(2)}-\frac{2}{3} \lambda_{p}\left(x_{i+(1 / 2)}^{(2)}, t^{n}+\frac{1}{2} \tau_{l} \Delta t\right) \tau_{l} \Delta t,
\end{aligned}
$$

where $p=1,2, \ldots, m$. The semi-Lagrangian finite different scheme for the system (21) is taken as

$$
\begin{aligned}
& \mathbf{u}_{i}^{n+1} \\
&=\mathbf{u}_{i}^{n}-\Delta t \sum_{l=1}^{3} \rho_{l}\left(c \left(\left(\widehat{\mathbf{f}}\left(\mathbf{u}\left(x_{i+(3 / 2)}^{(3)}, t^{n}\right)\right)\right.\right.\right. \\
&\left.\left.-\widehat{\mathbf{f}}\left(\mathbf{u}\left(x_{i-(3 / 2)}^{(3)}, t^{n}\right)\right)\right)(3 \Delta x)^{-1}\right) \\
&+d\left(\left(\widehat{\mathbf{f}}\left(\mathbf{u}\left(x_{i+(1 / 2)}^{(3)}, t^{n}\right)\right)\right.\right. \\
&\left.\left.\left.\quad-\widehat{\mathbf{f}}\left(\mathbf{u}\left(x_{i-(1 / 2)}^{(3)}, t^{n}\right)\right)\right)(\Delta x)^{-1}\right)\right),
\end{aligned}
$$

where $c$ and $d$ are the corresponding compact coefficients determined by (41). The WENO interpolation is provided in detail in Section 4.3.
4.3. Mapped Fifth Order WENO Interpolation. In the subsection, we shall first consider the case when $\lambda_{p} \geq 0$, after that we will consider the case when $\lambda_{p}<0$. The details of the method are omitted and necessary formulas are given in the following.

Case $1\left(\lambda_{p} \geq 0\right)$. In the fifth order interpolation, we always use the three points stencils $\left\{f_{i-2}^{n}, f_{i-1}^{n}, f_{i}^{n}\right\},\left\{f_{i-1}^{n}, f_{i}^{n}, f_{i+1}^{n}\right\}$, and $\left\{f_{i}^{n}, f_{i+1}^{n}, f_{i+2}^{n}\right\}$ to interpolate polynomials $p_{r}(x)(r=$ $0,1,2)$. Then we use the stencil $\left\{f_{i-2}^{n}, f_{i-1}^{n}, f_{i}^{n}, f_{i+1}^{n}, f_{i+2}^{n}\right\}$ to interpolate polynomial $q(x)$. We have the following scheme:

$$
\begin{aligned}
& p_{0}\left(x_{i+(1 / 2)}^{(3)}\right)=\left(\frac{3}{8}-\xi_{i}+\frac{\xi_{i}^{2}}{2}\right) f_{i-2}^{n}+\left(-\frac{10}{8}+3 \xi_{i}-\xi_{i}^{2}\right) f_{i-1}^{n} \\
& +\left(\frac{15}{8}-2 \xi_{i}+\frac{\xi_{i}^{2}}{2}\right) f_{i}^{n} \\
& p_{1}\left(x_{i+(1 / 2)}^{(3)}\right)=\left(-\frac{1}{8}+\frac{\xi_{i}^{2}}{2}\right) f_{i-1}^{n}+\left(\frac{6}{8}+\xi_{i}-\xi_{i}^{2}\right) f_{i}^{n} \\
& +\left(\frac{3}{8}-\xi_{i}+\frac{\xi_{i}^{2}}{2}\right) f_{i+1}^{n} \\
& p_{2}\left(x_{i+(1 / 2)}^{(3)}\right)=\left(\frac{3}{8}+\xi_{i}+\frac{\xi_{i}^{2}}{2}\right) f_{i}^{n}+\left(\frac{6}{8}-\xi_{i}-\xi_{i}^{2}\right) f_{i+1}^{n} \\
& +\left(-\frac{1}{8}+\frac{\xi_{i}^{2}}{2}\right) f_{i+2}^{n} \\
& q\left(x_{i+(1 / 2)}^{(3)}\right)=\left(\frac{3}{128}-\frac{5 \xi_{i}^{2}}{48}+\frac{\xi_{i}^{4}}{24}\right) f_{i-2}^{n} \\
& +\left(-\frac{5}{32}-\frac{\xi_{i}}{24}+\frac{2 \xi_{i}^{2}}{3}+\frac{\xi_{i}^{3}}{6}-\frac{\xi_{i}^{4}}{6}\right) f_{i-1}^{n} \\
& +\left(\frac{45}{64}+\frac{9 \xi_{i}}{8}-\frac{7 \xi_{i}^{2}}{8}-\frac{\xi_{i}^{3}}{2}+\frac{\xi_{i}^{4}}{4}\right) f_{i}^{n} \\
& +\left(\frac{15}{32}-\frac{27 \xi_{i}}{24}+\frac{\xi_{i}^{2}}{6}+\frac{\xi_{i}^{3}}{2}-\frac{\xi_{i}^{4}}{6}\right) f_{i+1}^{n} \\
& +\left(-\frac{5}{128}+\frac{\xi_{i}}{24}+\frac{7 \xi_{i}^{2}}{48}-\frac{\xi_{i}^{3}}{6}+\frac{\xi_{i}^{4}}{24}\right) f_{i+2}^{n},
\end{aligned}
$$

and $d_{0}, d_{1}, d_{2}$, are the corresponding linear weights given by

$$
\begin{aligned}
& d_{0}=\frac{1}{16}+\frac{\xi_{i}}{6}+\frac{\xi_{i}^{2}}{12} \\
& d_{1}=\frac{10}{16}+\frac{\xi_{i}}{6}-\frac{\xi_{i}^{2}}{6} \\
& d_{2}=\frac{5}{16}-\frac{\xi_{i}}{3}+\frac{\xi_{i}^{2}}{12} .
\end{aligned}
$$


The smoothness indicators are taken on the following form:

$$
\begin{aligned}
& \beta_{0}=\frac{13}{12}\left(f_{i-2}^{n}-2 f_{i-1}^{n}+f_{i}^{n}\right)^{2}+\frac{1}{4}\left(f_{i-2}^{n}-4 f_{i-1}^{n}+3 f_{i}^{n}\right)^{2}, \\
& \beta_{1}=\frac{13}{12}\left(f_{i-1}^{n}-2 f_{i}^{n}+f_{i+1}^{n}\right)^{2}+\frac{1}{4}\left(f_{i-1}^{n}-f_{i+1}^{n}\right)^{2}, \\
& \beta_{2}=\frac{13}{12}\left(f_{i}^{n}-2 f_{i+1}^{n}+f_{i+2}^{n}\right)^{2}+\frac{1}{4}\left(3 f_{i}^{n}-4 f_{i+1}^{n}+f_{i+2}^{n}\right)^{2} .
\end{aligned}
$$

The nonlinear weights satisfy

$$
\omega_{r}=\frac{\alpha_{r}}{\sum_{s=0}^{2} \alpha_{s}}, \quad \alpha_{r}=\frac{d_{r}}{\left(\epsilon+\beta_{r}\right)^{2}}, \quad r=0,1,2
$$

The mapped nonlinear weights $\omega_{r}^{(M)}$ are then computed from

$$
\omega_{r}^{(M)}=\frac{\alpha_{r}^{*}}{\sum_{s=0}^{2} \alpha_{s}^{*}}, \quad \alpha_{r}^{*}=m_{r}\left(\omega_{r}\right), \quad r=0,1,2 .
$$

Here $\epsilon>0$ is to avoid the denominator becoming zero; we use the value $\varepsilon=10^{-40}$ in our numerical tests. The numerical flux is computed by the following fifth order mapped WENO scheme with the semi-Lagrangian compact finite difference scheme:

$$
\left(\widehat{f}_{i+(1 / 2)}^{(3)}\right)^{-}=\sum_{r=0}^{2} \omega_{r}^{(M)} p_{r}\left(x_{i+(1 / 2)}^{(3)}\right) .
$$

Case $2\left(\lambda_{p}<0\right)$. We use the three points stencils $\left\{f_{i+3}^{n}, f_{i+2}^{n}, f_{i+1}^{n}\right\},\left\{f_{i+2}^{n}, f_{i+1}^{n}, f_{i}^{n}\right\}$, and $\left\{f_{i+1}^{n}, f_{i}^{n}, f_{i-1}^{n}\right\}$ and the stencil $\left\{f_{i+3}^{n}, f_{i+2}^{n}, f_{i+1}^{n}, f_{i}^{n}, f_{i-1}^{n}\right\}$ to build the scheme. We have

$$
\begin{aligned}
p_{0}\left(x_{i+(1 / 2)}^{(3)}\right)= & \left(\frac{3}{8}+\xi_{i}+\frac{\xi_{i}^{2}}{2}\right) f_{i+3}^{n}+\left(-\frac{10}{8}-3 \xi_{i}-\xi_{i}^{2}\right) f_{i+2}^{n} \\
& +\left(\frac{15}{8}+2 \xi_{i}+\frac{\xi_{i}^{2}}{2}\right) f_{i+1}^{n}, \\
p_{1}\left(x_{i+(1 / 2)}^{(3)}\right)= & \left(-\frac{1}{8}+\frac{\xi_{i}^{2}}{2}\right) f_{i+2}^{n}+\left(\frac{6}{8}-\xi_{i}-\xi_{i}^{2}\right) f_{i+1}^{n} \\
& +\left(\frac{3}{8}+\xi_{i}+\frac{\xi_{i}^{2}}{2}\right) f_{i}^{n}, \\
& +\left(-\frac{3}{8}+\frac{\xi_{i}^{2}}{2}\right) f_{i-1}^{n},
\end{aligned}
$$

$$
\begin{aligned}
q\left(x_{i+(1 / 2)}^{(3)}\right)= & \left(\frac{3}{128}-\frac{5 \xi_{i}^{2}}{48}+\frac{\xi_{i}^{4}}{24}\right) f_{i+3}^{n} \\
& +\left(-\frac{5}{32}+\frac{\xi_{i}}{24}+\frac{2 \xi_{i}^{2}}{3}-\frac{\xi_{i}^{3}}{6}-\frac{\xi_{i}^{4}}{6}\right) f_{i+2}^{n} \\
& +\left(\frac{45}{64}-\frac{9 \xi_{i}}{8}-\frac{7 \xi_{i}^{2}}{8}+\frac{\xi_{i}^{3}}{2}+\frac{\xi_{i}^{4}}{4}\right) f_{i+1}^{n} \\
& +\left(\frac{15}{32}+\frac{27 \xi_{i}}{24}+\frac{\xi_{i}^{2}}{6}-\frac{\xi_{i}^{3}}{2}-\frac{\xi_{i}^{4}}{6}\right) f_{i}^{n} \\
& +\left(-\frac{5}{128}-\frac{\xi_{i}}{24}+\frac{7 \xi_{i}^{2}}{48}+\frac{\xi_{i}^{3}}{6}+\frac{\xi_{i}^{4}}{24}\right) f_{i-1}^{n}
\end{aligned}
$$

and the corresponding linear weights, which are denoted by $d_{0}, d_{1}, d_{2}$. In this case,

$$
\begin{aligned}
& d_{0}=\frac{1}{16}-\frac{\xi_{i}}{6}+\frac{\xi_{i}^{2}}{12}, \\
& d_{1}=\frac{10}{16}-\frac{\xi_{i}}{6}-\frac{\xi_{i}^{2}}{6}, \\
& d_{2}=\frac{5}{16}+\frac{\xi_{i}}{3}+\frac{\xi_{i}^{2}}{12} .
\end{aligned}
$$

The smoothness indicators are taken on the following form:

$$
\begin{aligned}
& \beta_{0}=\frac{13}{12}\left(f_{i+3}^{n}-2 f_{i+2}^{n}+f_{i+1}^{n}\right)^{2}+\frac{1}{4}\left(f_{i+3}^{n}-4 f_{i+2}^{n}+3 f_{i+1}^{n}\right)^{2}, \\
& \beta_{1}=\frac{13}{12}\left(f_{i+2}^{n}-2 f_{i+1}^{n}+f_{i+1}^{n}\right)^{2}+\frac{1}{4}\left(f_{i+2}^{n}-f_{i}^{n}\right)^{2}, \\
& \beta_{2}=\frac{13}{12}\left(f_{i+1}^{n}-2 f_{i}^{n}+f_{i-1}^{n}\right)^{2}+\frac{1}{4}\left(3 f_{i+1}^{n}-4 f_{i}^{n}+f_{i-1}^{n}\right)^{2} .
\end{aligned}
$$

The numerical flux is reconstructed by the following fifth order mapped WENO scheme:

$$
\left(\widehat{f}_{i+(1 / 2)}^{(3)}\right)^{-}=\sum_{r=0}^{2} \omega_{r}^{(M)} p_{r}\left(x_{i+(1 / 2)}^{(3)}\right) .
$$

4.4. Boundary Conditions. It should be pointed out that the derived-boundary and near-boundary schemes are significant for the fifth-order mapped M-C-SL-FD WENO schemes. The boundary conditions are crucial for the order of accuracy, and they affect the stability characteristics of the scheme. In 
this study, the fourth-order accuracy boundary programme of Zhang et al. [9] was used as follows:

$$
\begin{gathered}
f_{0}^{\prime}+3 f_{1}^{\prime}=\frac{1}{6 \Delta x}\left(-17 f_{0}^{\prime}+9 f_{1}^{\prime}+9 f_{2}^{\prime}-f_{3}^{\prime}\right), \\
f_{0}^{\prime}+4 f_{1}^{\prime}+f_{2}^{\prime}=\frac{1}{\Delta x}\left(-3 f_{0}^{\prime}+3 f_{2}^{\prime}\right), \\
f_{N}^{\prime}+3 f_{N-1}^{\prime}=\frac{1}{6 \Delta x}\left(-17 f_{N-3}^{\prime}+9 f_{N-2}^{\prime}+9 f_{N-1}^{\prime}+17 f_{N}^{\prime}\right), \\
f_{N}^{\prime}+4 f_{N-1}^{\prime}+f_{N-2}^{\prime}=\frac{1}{\Delta x}\left(-3 f_{N}^{\prime}+3 f_{N-2}^{\prime}\right) .
\end{gathered}
$$

It should be observed that the accuracy of the boundary conditions is fourth-order, but that does not reduce the fifthorder accuracy of the M-C-SL-FD WENO schemes; that is, fourth-order accuracy boundary conditions maintain fifthorder accuracy and stability of the scheme proposed in this study.

Remark 1. Negative linear weights in a WENO scheme produce oscillations and instability. Shi et al. [30] have dealt with this problem using a simple and effective splitting technique which obviates the need to remove the negative weights and retains the stability and accuracy of the scheme.

Remark 2. If the systems under consideration have $m$ characteristic variables, such as

$$
\lambda_{1}(u), \lambda_{2}(u), \ldots, \lambda_{m}(u),
$$

Ii and Xiao [28] have produced a method incorporating a decoupled system of characteristic variables and Riemann invariants and then solved the linear systems for primitive variables along the characteristic curves, which is the approach adopted in this paper.

Remark 3. When the CFL number is large enough to make $x_{i+(1 / 2)}-x_{i+(1 / 2)}^{(3)}>\Delta x_{i}$, Qiu and Shu have proposed a large time-step evolution method to handle this condition [19].

\section{Numerical Examples}

Massive numerical experiments to assess the performance of the M-SL-FV WENO 5 and M-C-SL-FD WENO 5 schemes are described in this section. We present the results of our numerical tests for scalar and for system of conservation laws. If in the following examples the characteristics were variable, we adopted the third-order TVD RK method. The mapped semi-Lagrangian WENO 5 schemes and semi-Lagrangian WENO 5 schemes allow a weaker CFL condition. In the examples, the CFL number is taken as 5.9 for the linear advection problem and Burger's equation and 9.9 for the onedimensional Euler equations. A CFL number of 0.2 is chosen for the FV WENO 5 and FD WENO 5 schemes. All solutions are computed using uniform meshes. In all of the simulations, the mapped semi-Lagrangian schemes use $\varepsilon=10^{-40}$. For the semi-Lagrangian, FV and FD WENO schemes use $\varepsilon=10^{-6}$.

\subsection{Accuracy Test}

Example 1 (the linear advection problem). We check the order of accuracy of M-SL-FV, M-C-SL-FD, SL-FV, C-SL$\mathrm{FD}, \mathrm{FV}$, and FD WENO 5 methods for the linear advection problem

$$
u_{t}+u_{x}=0
$$

The initial condition is given by $u(x, 0)=\sin (\pi x-\sin (\pi x) / \pi)$, the investigative domain is $[-1,1]$, and the boundary conditions are periodic. The exact solution of this problem is given by

$$
u(x, t)=\sin \left(\pi(x-t)-\frac{\sin (\pi(x-t))}{\pi}\right) .
$$

The norm of the error is computed at time $t=0.5$ and $\mathrm{CFL}=5.9$. The errors and numerical orders of accuracy for the M-SL-FV WENO 5 scheme and M-C-SL-FD WENO 5 scheme are shown in Table 1. The convergence results of the scheme without the mapped weights are listed in Table 2. The convergence rates of Tables 1 and 2 show that M-SLFV WENO 5 and M-C-SL-FD WENO 5 with $\varepsilon=10^{-40}$ schemes can reach fifth-order accuracy. In fact, the error is third-order accuracy in the $L^{\infty}$ norm with $\varepsilon=10^{-6}$ as shown in Table 2. The $L^{1}$ and $L^{\infty}$ errors of the FV and FD WENO 5 methods are shown in Table 3. Tables 1 and 3 show the orders of the accuracy for the M-SL-FV, M-C-SL-FD, $\mathrm{FV}$, and FD WENO 5 methods. We see that the FV and FD WENO 5 methods do not achieve fifth-order accuracy for the linear advection problem. It is important to point out that the FV and FD methods are worse than the M-SL-FV and M-C-SL-FD methods for the linear advection problem. So we can confirm that the mapped weights scheme gives a good approximation to the exact solution, and the M-SL-FV WENO 5 and M-C-SL-FD WENO 5 methods perform well for the linear advection problem.

\subsection{Numerical Tests}

Example 2 (Burgers equation). In order to demonstrate the stability of the M-SL-FV WENO 5 scheme and M-C-SL-FD WENO 5 scheme, the nonlinear scalar Burgers equation is

$$
u_{t}+\left(\frac{u^{2}}{2}\right)_{x}=0
$$

We take $u(x, 0)=0.5+\sin (\pi x)$ as the initial condition. The domain of the equation in this study is taken as $[0,2]$, and $\mathrm{CFL}=5.9$ with periodic boundary conditions. We show the results at $t=0.5 / \pi$ and $t=1.5 / \pi$ with $N=50$. The solution remains smooth at $t=0.5 / \pi$, but a shock wave is produced at $t=1.5 / \pi$. The outcomes of the M-SL-FV WENO 5 and M-CSL-FD WENO 5 schemes at time $t=0.5 / \pi$ and $t=1.5 / \pi$ are shown in Figures 1 and 2, respectively. The results are seen to be reasonable; both the M-SL-FV WENO 5 and M-C-SL-FD WENO 5 schemes solve the numerical oscillations very well at $t=1.5 / \pi$. 
TABLE 1: Left: M-SL-FV WENO 5 scheme, right: M-C-SL-FD WENO 5 scheme for Example 1 on a uniform mesh of $N$ cells at $t=0.5$, CFL $=$ 5.9 , and $\varepsilon=10^{-40}$.

\begin{tabular}{|c|c|c|c|c|c|c|c|c|}
\hline \multirow{2}{*}{$N$} & \multicolumn{4}{|c|}{ M-SL-FV WENO 5} & \multicolumn{4}{|c|}{ M-C-SL-FD WENO 5} \\
\hline & $L^{1}$ error & Order & $L^{\infty}$ error & Order & $L^{1}$ error & Order & $L^{\infty}$ error & Order \\
\hline 10 & $1.00 E-02$ & - & $2.03 E-02$ & - & $7.79 E-03$ & - & $2.45 E-02$ & - \\
\hline 20 & $4.66 E-04$ & 4.42 & $1.43 E-03$ & 3.83 & $3.76 E-04$ & 4.37 & $1.30 E-03$ & 4.24 \\
\hline 40 & $1.72 E-05$ & 4.76 & $5.25 E-05$ & 4.77 & $9.46 E-06$ & 5.31 & $3.72 E-05$ & 5.13 \\
\hline 80 & $5.68 E-07$ & 4.92 & $1.62 E-06$ & 5.02 & $3.17 E-07$ & 4.90 & $1.15 E-06$ & 5.02 \\
\hline 160 & $1.78 E-08$ & 5.00 & $5.09 E-08$ & 4.99 & $9.32 E-09$ & 5.09 & $3.25 E-08$ & 5.15 \\
\hline
\end{tabular}

TABLE 2: Left: SL-FV WENO 5 scheme, right: C-SL-FD WENO 5 scheme for Example 1 on a uniform mesh of $N$ cells at $t=0.5$, CFL $=5.9$, and $\varepsilon=10^{-6}$.

\begin{tabular}{|c|c|c|c|c|c|c|c|c|}
\hline \multirow{2}{*}{$N$} & \multicolumn{4}{|c|}{ SL-FV WENO 5} & \multicolumn{4}{|c|}{ C-SL-FD WENO 5} \\
\hline & $L^{1}$ error & Order & $L^{\infty}$ error & Order & $L^{1}$ error & Order & $L^{\infty}$ error & Order \\
\hline 10 & $1.83 E-02$ & - & $4.41 E-02$ & - & $1.21 E-02$ & - & $3.91 E-02$ & - \\
\hline 20 & $1.97 E-03$ & 3.22 & $4.62 E-03$ & 3.25 & $1.76 E-03$ & 2.78 & $4.07 E-03$ & 3.26 \\
\hline 40 & $1.07 E-04$ & 4.20 & $3.78 E-04$ & 3.61 & $7.69 E-05$ & 4.52 & $3.26 E-04$ & 3.64 \\
\hline 80 & $4.68 E-06$ & 4.52 & $2.96 E-05$ & 3.67 & $3.46 E-06$ & 4.47 & $2.56 E-05$ & 3.67 \\
\hline 160 & $1.96 E-07$ & 4.58 & $2.46 E-06$ & 3.59 & $1.65 E-07$ & 4.39 & $2.36 E-06$ & 3.44 \\
\hline
\end{tabular}

TABLE 3: Left: FV WENO 5 scheme, right: FD WENO 5 scheme for Example 1 on a uniform mesh of $N$ cells at $t=0.5$, CFL $=0.2$, and $\varepsilon=10^{-6}$.

\begin{tabular}{|c|c|c|c|c|c|c|c|c|}
\hline \multirow{2}{*}{$N$} & \multicolumn{4}{|c|}{ FV WENO 5} & \multicolumn{4}{|c|}{ FD WENO 5} \\
\hline & $L^{1}$ error & Order & $L^{\infty}$ error & Order & $L^{1}$ error & Order & $L^{\infty}$ error & Order \\
\hline 10 & $1.84 E-02$ & - & $4.44 E-02$ & - & $1.99 E-02$ & - & $4.77 E-02$ & - \\
\hline 20 & $1.93 E-03$ & 3.25 & $4.62 E-03$ & 3.26 & $2.02 E-03$ & 3.30 & $4.73 E-03$ & 3.33 \\
\hline 40 & $1.07 E-04$ & 4.17 & $3.78 E-04$ & 3.61 & $1.08 E-04$ & 4.23 & $3.83 E-04$ & 3.63 \\
\hline 80 & $4.72 E-06$ & 4.50 & $3.00 E-05$ & 3.66 & $4.75 E-06$ & 4.51 & $3.02 E-05$ & 3.66 \\
\hline 160 & $2.13 E-07$ & 4.47 & $2.76 E-06$ & 3.44 & $2.14 E-07$ & 4.47 & $2.77 E-06$ & 3.45 \\
\hline
\end{tabular}

Now, we begin to study the one-dimensional Euler system, written as

$$
\mathbf{u}_{t}+\mathbf{f}(\mathbf{u})_{x}=0
$$

where

$$
\mathbf{u}=\left(\begin{array}{c}
\rho \\
\rho v \\
E
\end{array}\right), \quad \mathbf{f}(\mathbf{u})=\left(\begin{array}{c}
\rho v \\
\rho v^{2}+p \\
v(E+p)
\end{array}\right)
$$

Here $\rho, v, E$, and $p$ are the density, velocity, total energy, and pressure, respectively. The equation of state has the following form:

$$
E=\frac{p}{\gamma-1}+\frac{1}{2} \rho v^{2}
$$

and the ratio of specific heat $\gamma=1.4$.

Example 3 (the Sod problem). The sod problem is the Euler equation (61), specified by the following initial conditions:

$$
(\rho, v, p)= \begin{cases}(1,0,1) & x \leq 0 \\ (0.125,0,0.1) & x>0 .\end{cases}
$$

Setting $[-5,5]$ as the computational domain of this problem, we show the results at $t=1.3$ and CFL $=9.9$ with periodic boundary conditions. In Figure 3, the outcomes of the MSL-FV WENO 5 and M-C-SL-FD WENO 5 schemes with a uniform grid of 200 points are shown. It is important to point out that the shock and contact discontinuity are correctly captured by both schemes.

Example 4 (The Lax problem). We consider the lax problem, which is the Euler equation (61) and has the following starting conditions:

$$
(\rho, v, p)= \begin{cases}(0.445,0.698,3.528) & x \leq 0 \\ (0.5,0,0.571) & x>0\end{cases}
$$

Setting $[-5,5]$ as the investigative domain of this problem, the solution is up to $t=1.3$ and CFL $=9.9$ with periodic boundary conditions. In the example, the M-SL-FV WENO 5 scheme and M-C-SL-FD WENO 5 scheme are used to calculate the solution. The simulated density of the lax problem with a uniform gird of 200 points against the exact solution are displayed in Figure 4. It can be seen that the two schemes maintain the ENO property and have a good nonoscillatory resolution at the discontinuities. 


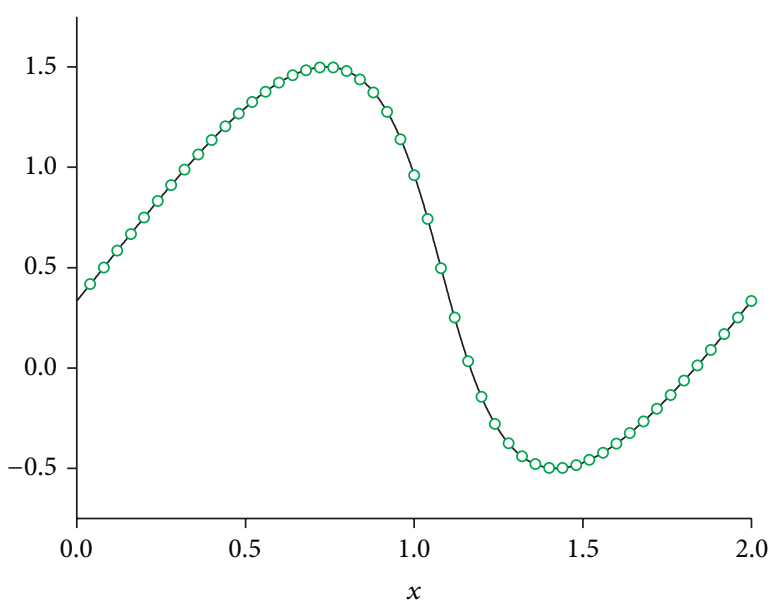

(a)

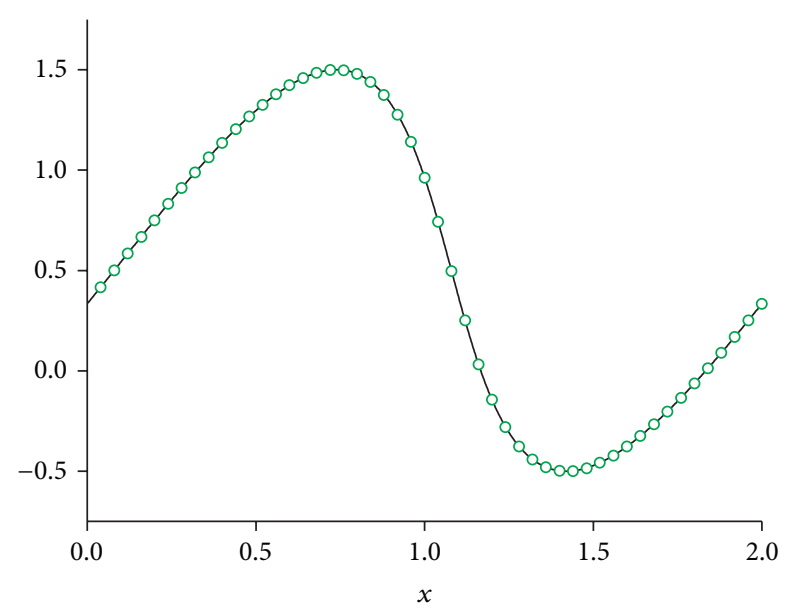

(b)

FIGURE 1: The numerical results of the Burgers equation at $t=0.5 / \pi$ with CFL $=5.9, N=50$; (a) M-SL-FV WENO 5 scheme; (b) M-C-SL-FD WENO 5 scheme; solid line: exact solution; circle: computed solution.

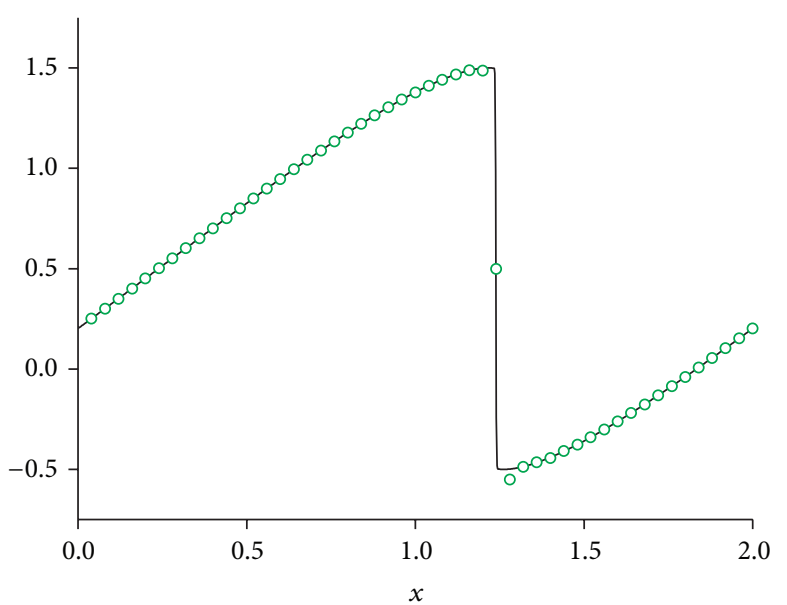

(a)

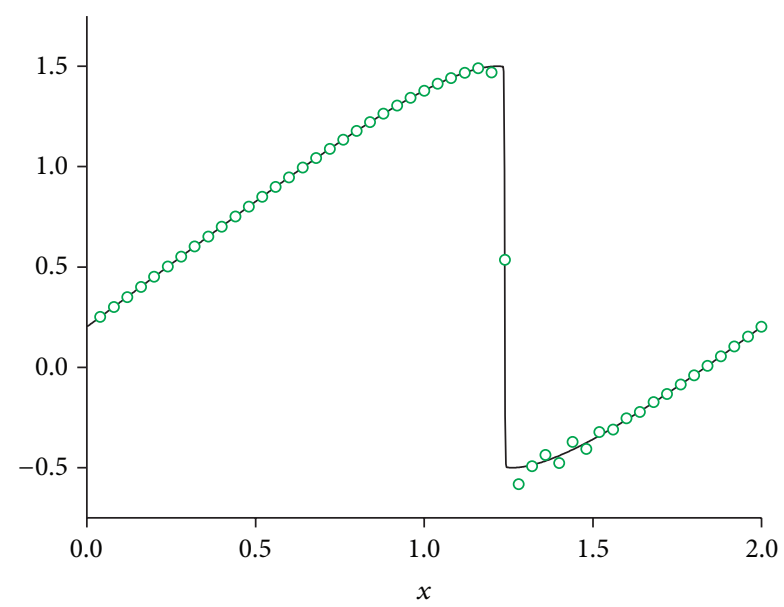

(b)

Figure 2: The numerical results of the Burgers equation at $t=1.5 / \pi$ with CFL $=5.9, N=50$; (a) M-SL-FV WENO 5 scheme; (b) M-C-SL-FD WENO 5 scheme; solid line: exact solution; circle: computed solution.

Example 5 (the shock-turbulence interaction problem). The shock-turbulence interaction problem is the Euler equation (61), specified by the following initial conditions:

$$
(\rho, v, p)= \begin{cases}(3.857143,2.629369,10.333333) & x \leq-4 \\ (1+\varepsilon \sin 5 x, 0,1) & x>-4\end{cases}
$$

Here we take $\varepsilon=0.2$. Setting $[-5,5]$ as the computational domain of this problem, the density is plotted at $t=1.8$ and $\mathrm{CFL}=9.9$ with periodic boundary conditions. The outcomes of the M-SL-FV WENO 5 scheme and M-C-SL-FD WENO 5 scheme are displayed in Figure 5. The figure shows that the two schemes can capture sharp, nonoscillatory shock near discontinuities.
Example 6 (the two interacting blast waves problem). In this example, we consider two interacting blast waves, which is defined by the Euler equation (61) and has the following starting conditions:

$$
(\rho, v, p)= \begin{cases}(1,0,1000) & 0 \leq x \leq 0.1 \\ (1,0,0.01) & 0.1 \leq x \leq 0.9 \\ (1,0,100) & \text { otherwise }\end{cases}
$$

Setting $[0,1]$ as the investigative domain of this problem, the solution is up to $t=0.038$ and CFL $=9.9$ with periodic boundary conditions. In the example, the simulated density of the two interacting blast waves problem are displayed for the M-SL-FV WENO 5 and M-C-SL-FD WENO 5 schemes in Figure 6 for a mesh of 400 cells. The figure shows clearly that 


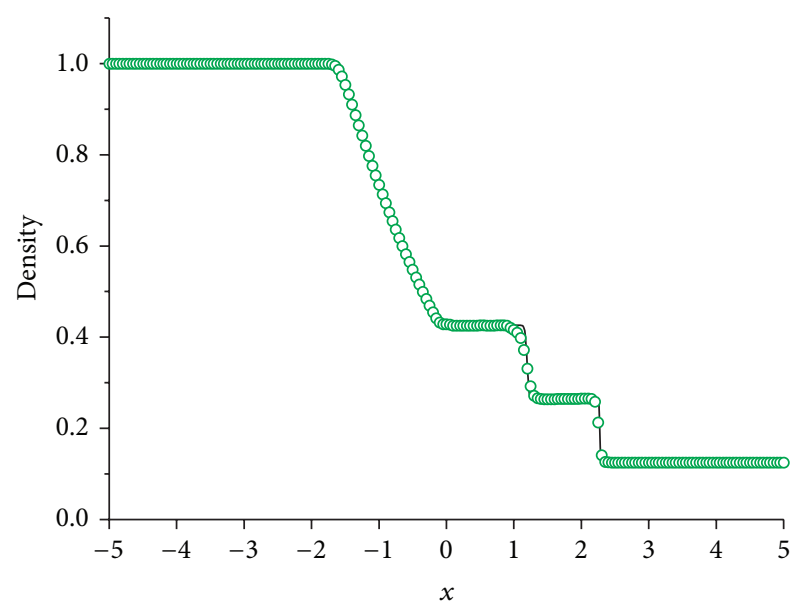

(a)

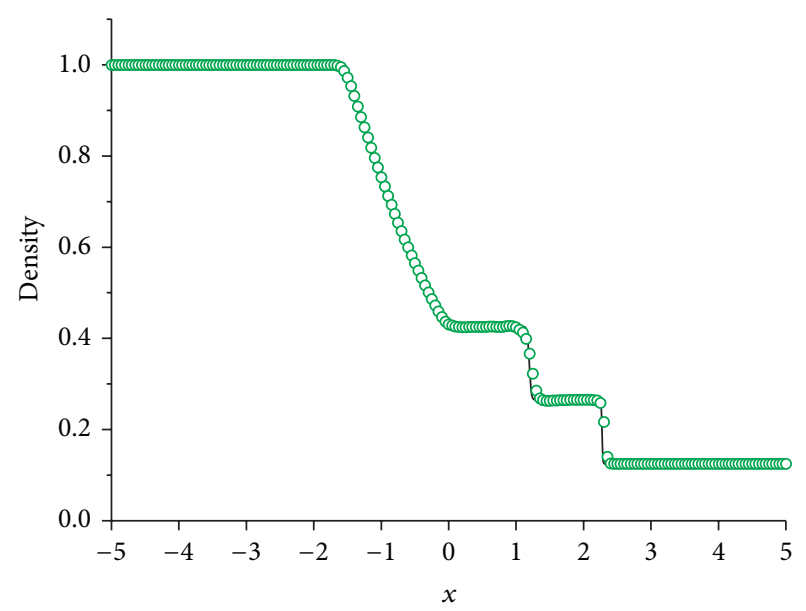

(b)

Figure 3: The numerical results of the Sod problem at $t=1.3$ with CFL $=9.9, N=200$; (a) M-SL-FV WENO 5 scheme; (b) M-C-SL-FD WENO 5 scheme; solid line: exact solution; circle: computed solution.

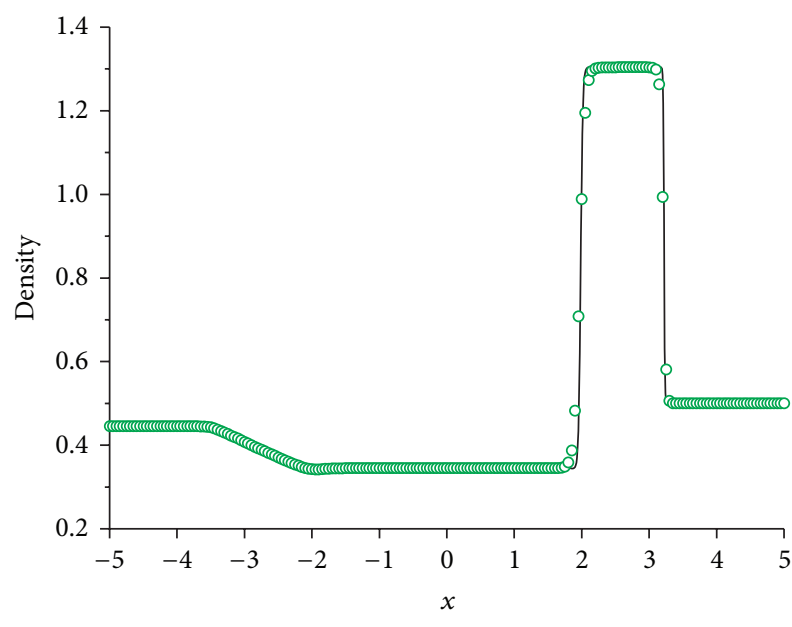

(a)

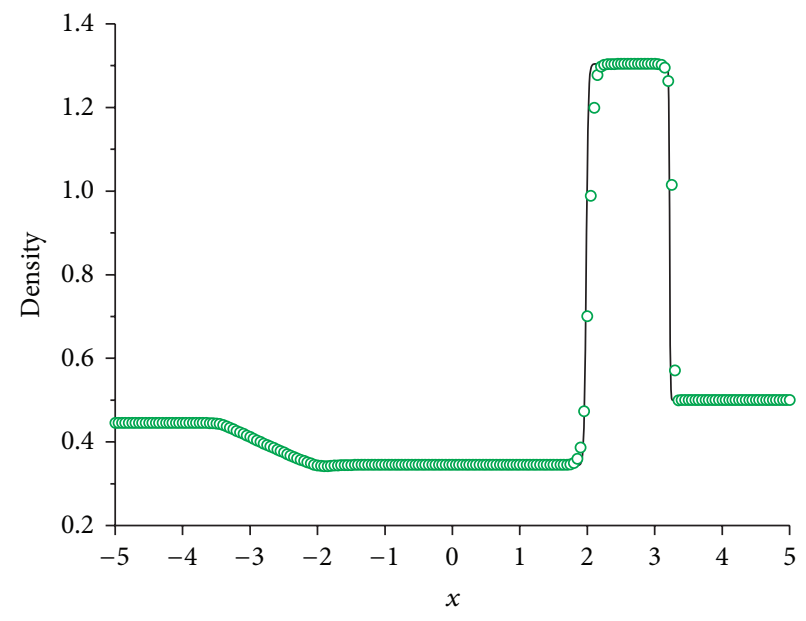

(b)

FIgURE 4: The numerical results of the lax problem at $t=1.3$ with CFL $=9.9, N=200$; (a) M-SL-FV WENO 5 scheme; (b) M-C-SL-FD WENO 5 scheme; solid line: exact solution; circle: computed solution.

the strong shock waves and contact discontinuities are best resolved by the M-SL-FV WENO 5 and M-C-SL-FD WENO 5 schemes. The numerical solutions are very satisfactory.

\section{Concluding Remarks}

Mapped semi-Lagrangian WENO methods are proposed in this paper. The M-SL-FV WENO 5 and M-C-SL-FD WENO 5 schemes were applied to one-dimensional hyperbolic conservation laws. Both schemes permit weaker CFL conditions, at the same time achieving fifth-order accuracy at certain smooth extrema and nonoscillatory shock near discontinuities. The numerical examples using the two schemes have demonstrated that they improve both accuracy and the ability to capture shock. The study of mapped semi-Lagrangian WENO schemes will be further expanded into the challenging areas of multidimensional nonlinear problems.

\section{Conflict of Interests}

The authors declare that there is no conflict of interests regarding the publication of this paper.

\section{Acknowledgments}

This work is partially supported by the National Science Foundation of China (11271100 and 11301113), the Ph.D. Programs Foundation of Ministry of Education of China 


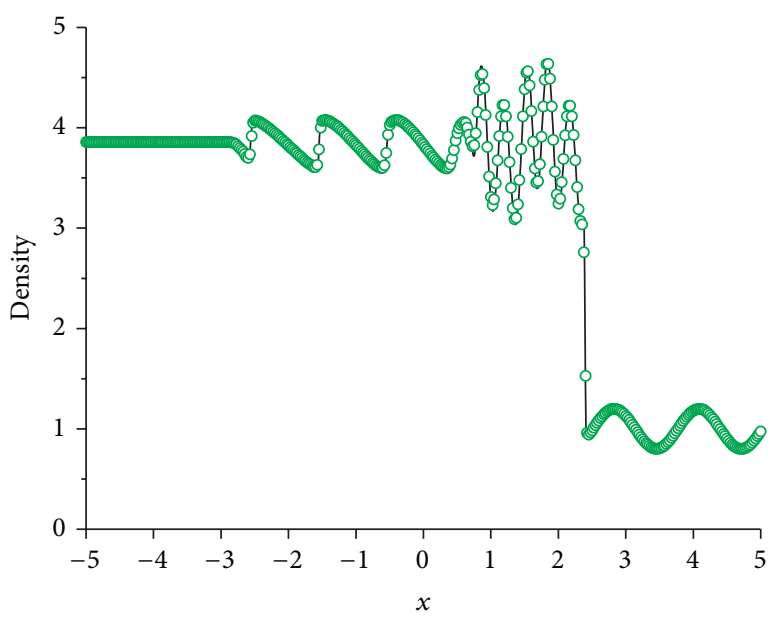

(a)

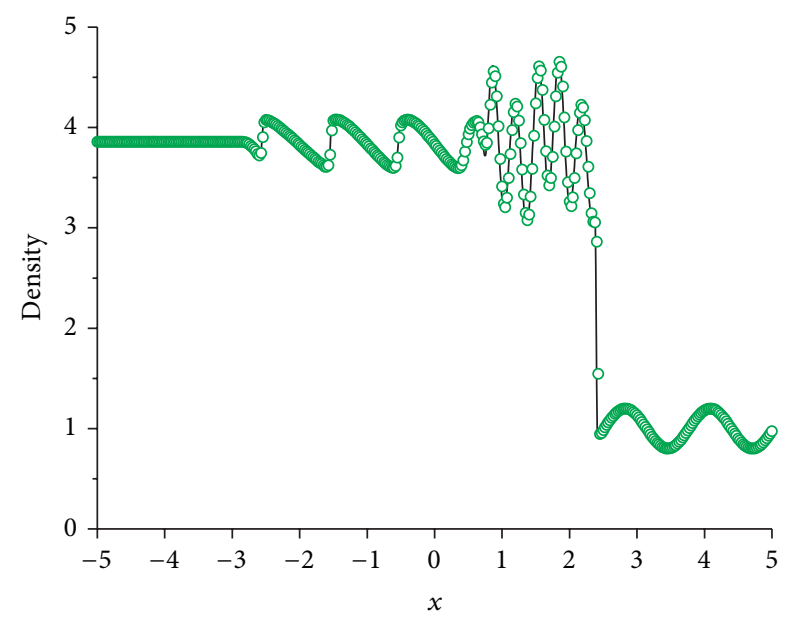

(b)

FIGURE 5: The numerical results of the shock-turbulence interaction problem at $t=1.8$ with CFL $=9.9, N=400$; (a) M-SL-FV WENO 5 scheme; (b) M-C-SL-FD WENO 5 scheme; solid line: exact solution; circle: computed solution.

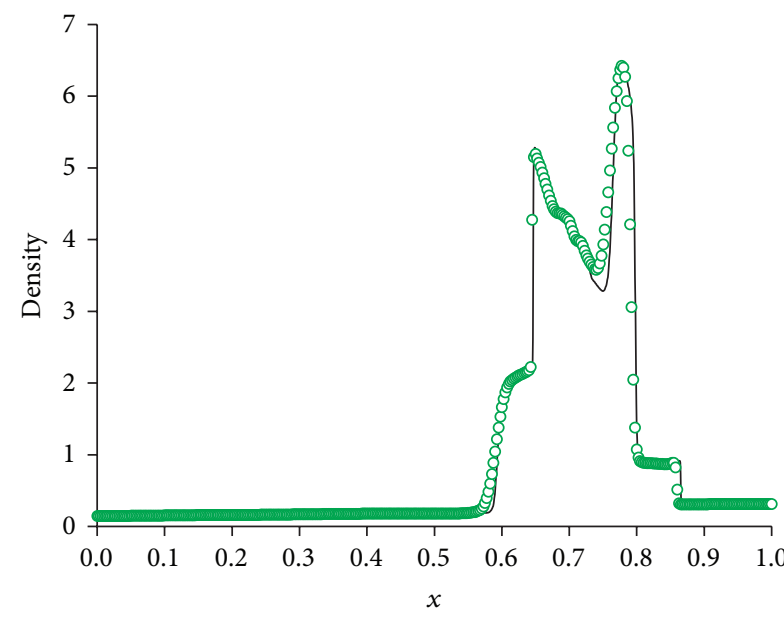

(a)

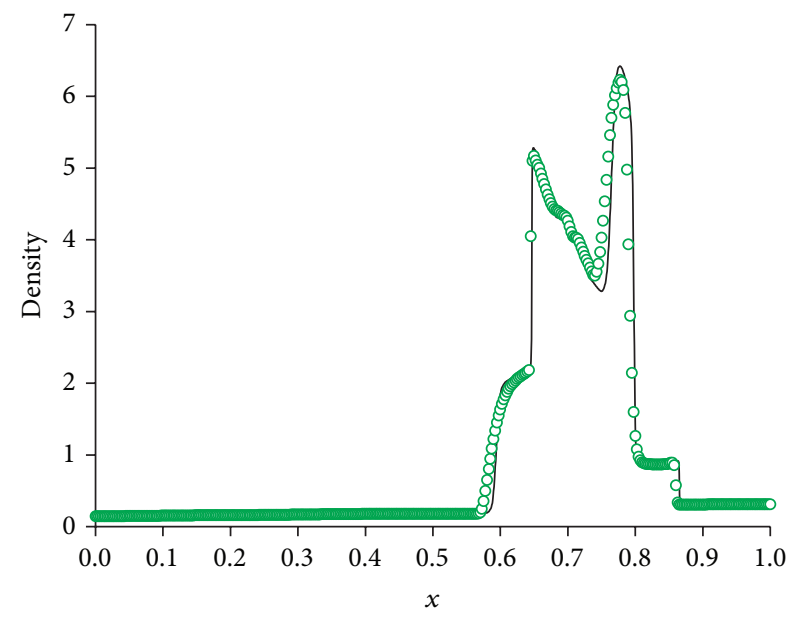

(b)

Figure 6: The numerical results of the two interacting blast waves problem at $t=0.038$ with CFL $=9.9, N=400$; (a) M-SL-FV WENO 5 scheme; (b) M-C-SL-FD WENO 5 scheme; solid line: exact solution; circle: computed solution.

(no. 20132302120057), the class General Financial Grant from the China Postdoctoral Science Foundation (Grant no. 2012M510933), the Fundamental Research Funds for the Central Universities (Grant nos. HIT. NSRIF. 2011003 and HIT. A. 201412), the Program for Innovation Research of Science in Harbin Institute of Technology (Grant no. PIRS OF HIT A201403), and Harbin Science and Technology Innovative Talents Project of Special Fund (2013RFXYJ044).

\section{References}

[1] A. K. Henrick, T. D. Aslam, and J. M. Powers, "Mapped weighted essentially non-oscillatory schemes: achieving optimal order near critical points," Journal of Computational Physics, vol. 207, no. 2, pp. 542-567, 2005.

[2] H. Feng, F. Hu, and R. Wang, "A new mapped weighted essentially non-oscillatory scheme," Journal of Scientific Computing, vol. 51, no. 2, pp. 449-473, 2012.

[3] S. Bryson and D. Levy, "Mapped WENO and weighted power ENO reconstructions in semi-discrete central schemes for Hamilton-Jacobi equations," Applied Numerical Mathematics, vol. 56, no. 9, pp. 1211-1224, 2006.

[4] R. Borges, M. Carmona, B. Costa, and W. S. Don, "An improved weighted essentially non-oscillatory scheme for hyperbolic conservation laws," Journal of Computational Physics, vol. 227, no. 6, pp. 3191-3211, 2008. 
[5] S. K. Lele, "Compact finite difference schemes with spectral-like resolution," Journal of Computational Physics, vol. 103, no. 1, pp. 16-42, 1992.

[6] X. Deng and H. Zhang, "Developing high-order weighted compact nonlinear schemes," Journal of Computational Physics, vol. 165, no. 1, pp. 22-44, 2000.

[7] L. Jiang, H. Shan, and C. Q. Liu, "Weighted compact scheme for shock capturing," International Journal of Computational Fluid Dynamics, vol. 15, no. 2, pp. 147-155, 2001.

[8] S. Pirozzoli, "Conservative hybrid compact-WENO schemes for shock-turbulence interaction," Journal of Computational Physics, vol. 178, no. 1, pp. 81-117, 2002.

[9] S. Zhang, S. Jiang, and C. Shu, "Development of nonlinear weighted compact schemes with increasingly higher order accuracy," Journal of Computational Physics, vol. 227, no. 15, pp. 7294-7321, 2008.

[10] C. W. Hirt, A. A. Amsden, and J. L. Cook, "An arbitrary Lagrangian-Eulerian computing method for all flow speeds," Journal of Computational Physics, vol. 135, no. 2, pp. 203-216, 1997.

[11] R. P. Fedkiw, T. Aslam, B. Merriman, and S. Osher, "A nonoscillatory Eulerian approach to interfaces in multimaterial flows (the ghost fluid method)," Journal of Computational Physics, vol. 152, no. 2, pp. 457-492, 1999.

[12] J. Cheng and C.-W. Shu, "A high order ENO conservative Lagrangian type scheme for the compressible Euler equations," Journal of Computational Physics, vol. 227, no. 2, pp. 1567-1596, 2007.

[13] W. Liu, J. Cheng, and C. Shu, "High order conservative Lagrangian schemes with LAX-Wendroff type time discretization for the compressible Euler equations," Journal of Computational Physics, vol. 228, no. 23, pp. 8872-8891, 2009.

[14] R. Acar, "Oscillation-free advection of interfaces with high order semi-Lagrangian schemes," Computers and Fluids, vol. 38, no. 1, pp. 137-159, 2009.

[15] R. D. Nair, J. S. Scroggs, and F. H. M. Semazzi, "A forwardtrajectory global semi-Lagrangian transport scheme," Journal of Computational Physics, vol. 190, no. 1, pp. 275-294, 2003.

[16] J. B. White and J. J. Dongarra, "High-performance highresolution semi-Lagrangian tracer transport on a sphere," Journal of Computational Physics, vol. 230, no. 17, pp. 6778-6799, 2011.

[17] N. Crouseilles, T. Respaud, and E. Sonnendrücker, "A forward semi-Lagrangian method for the numerical solution of the Vlasov equation," Computer Physics Communications, vol. 180, no. 10, pp. 1730-1745, 2009.

[18] N. Crouseilles, M. Mehrenberger, and E. Sonnendrücker, "Conservative semi-Lagrangian schemes for Vlasov equations," Journal of Computational Physics, vol. 229, no. 6, pp. 1927-1953, 2010.

[19] J. Qiu and C. Shu, "Conservative high order semi-Lagrangian finite difference WENO methods for advection in incompressible flow," Journal of Computational Physics, vol. 230, no. 4, pp. 863-889, 2011.

[20] J. Qiu and A. Christlieb, "A conservative high order semiLagrangian WENO method for the Vlasov equation," Journal of Computational Physics, vol. 229, no. 4, pp. 1130-1149, 2010.

[21] J. Qiu and C. Shu, "Conservative semi-Lagrangian finite difference WENO formulations with applications to the Vlasov equation," Communications in Computational Physics, vol. 10, no. 4, pp. 979-1000, 2011.

[22] J. Qiu and C. Shu, "Positivity preserving semi-Lagrangian discontinuous Galerkin formulation: theoretical analysis and application to the Vlasov-Poisson system," Journal of Computational Physics, vol. 230, no. 23, pp. 8386-8409, 2011.

[23] G.-S. Jiang and C.-W. Shu, "Efficient implementation of weighted ENO schemes," Journal of Computational Physics, vol. 126, no. 1, pp. 202-228, 1996.

[24] X. Liu, S. Osher, and T. Chan, "Weighted essentially non-oscillatory schemes," Journal of Computational Physics, vol. 115, no. 1, pp. 200-212, 1994.

[25] P. Montarnal and C. Shu, "Real gas computation using an energy relaxation method and high-order WENO schemes," Journal of Computational Physics, vol. 148, no. 1, pp. 59-80, 1999.

[26] J. Zhu, J. Qiu, T. Liu, and B. C. Khoo, "RKDG methods and WENO type limiters and conservative interfacial procedure for one-dimensional compressible multi-medium flow simulations," Applied Numerical Mathematics, vol. 61, no. 4, pp. 554580, 2011.

[27] C. Shu, "High order weighted essentially nonoscillatory schemes for convection dominated problems," SIAM Review, vol. 51, no. 1, pp. 82-126, 2009.

[28] S. Ii and F. Xiao, "CIP/multi-moment finite volume method for Eul er equations: a semi-Lagrangian characteristic formulation," Journal of Computational Physics, vol. 222, no. 2, pp. 849-871, 2007.

[29] C. W. Shu, "Total-variation-diminishing time discretizations," SIAM Journal on Scientific and Statistical Computing, vol. 9, no. 6, pp. 1073-1084, 1988.

[30] J. Shi, C. Hu, and C. Shu, "A technique of treating negative weights in WENO schemes," Journal of Computational Physics, vol. 175, no. 1, pp. 108-127, 2002. 


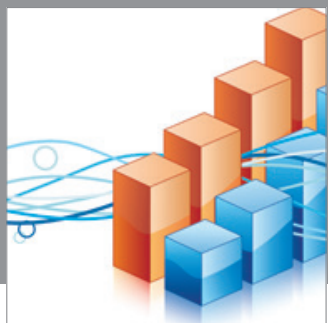

Advances in

Operations Research

mansans

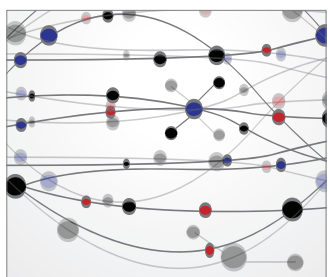

The Scientific World Journal
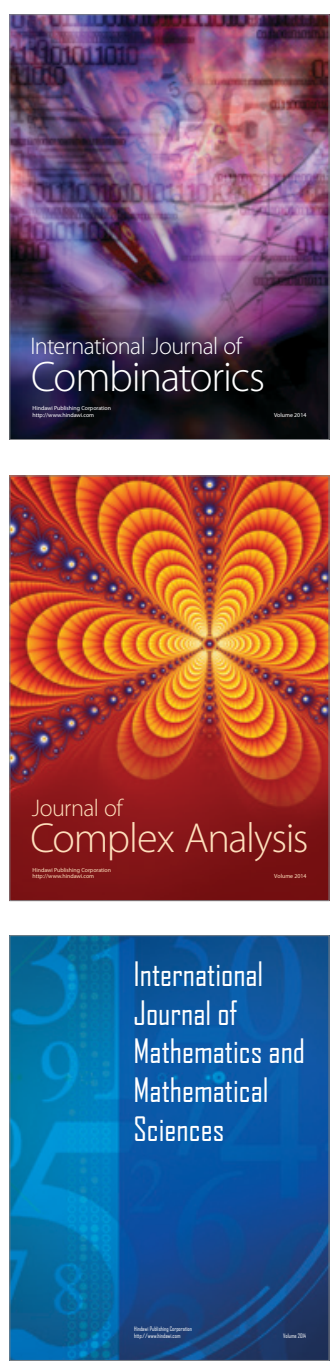
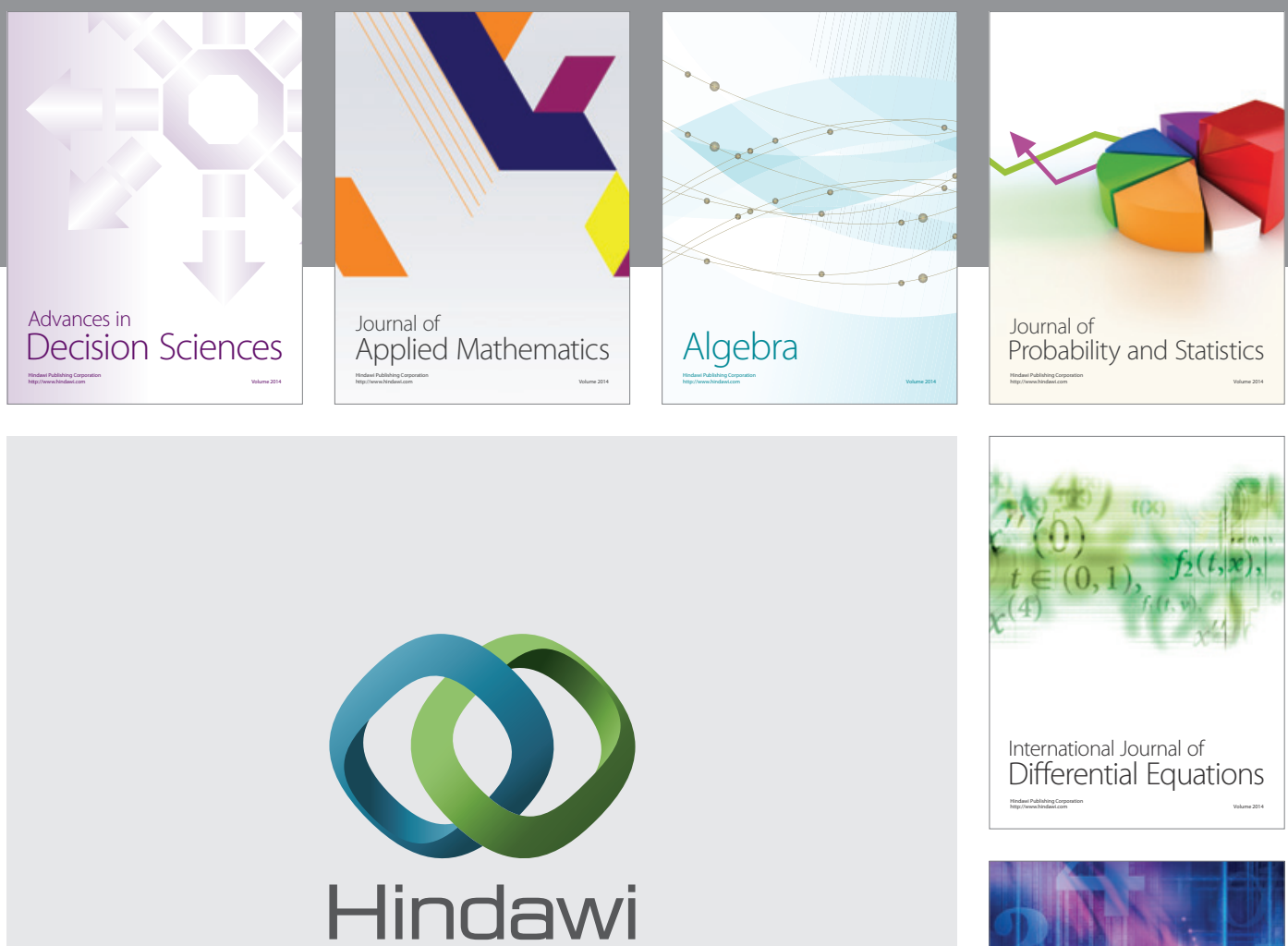

Submit your manuscripts at http://www.hindawi.com
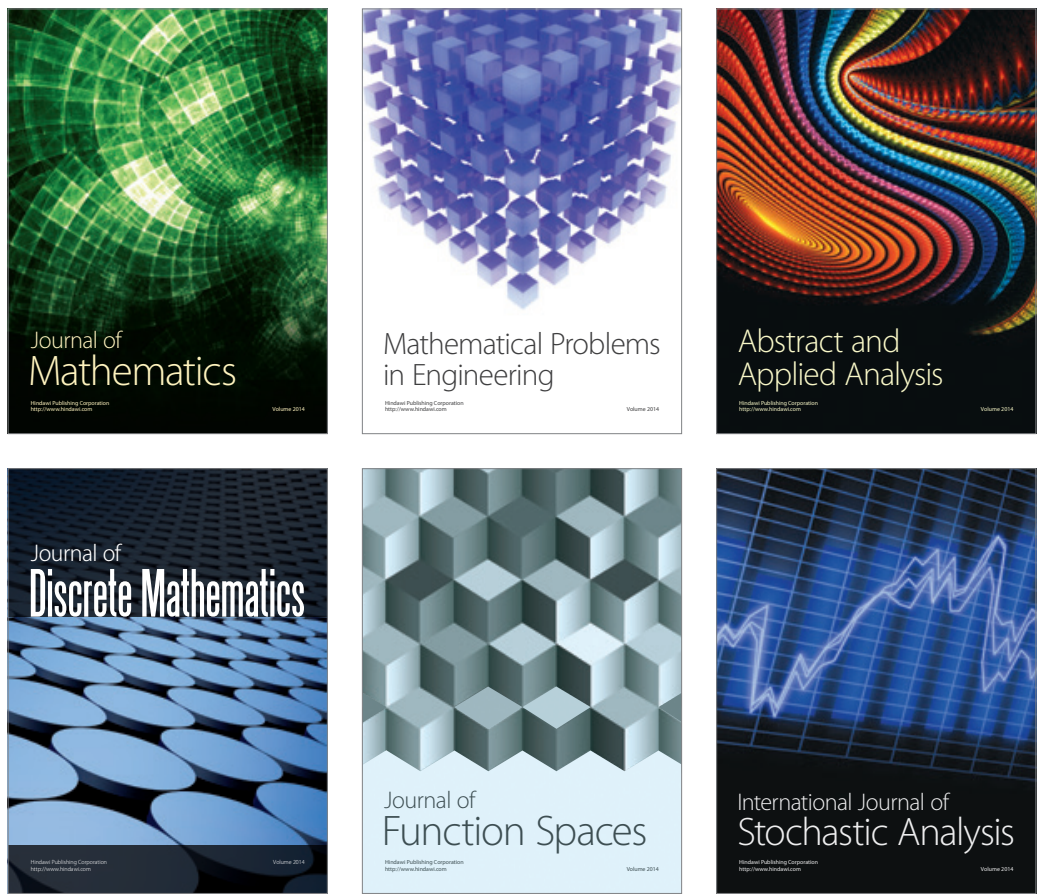

Journal of

Function Spaces

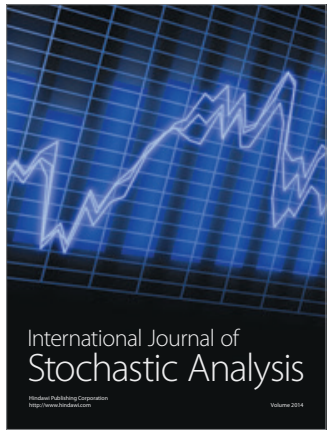

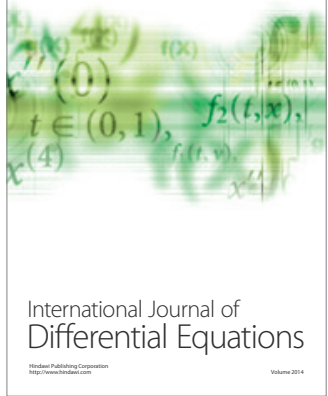
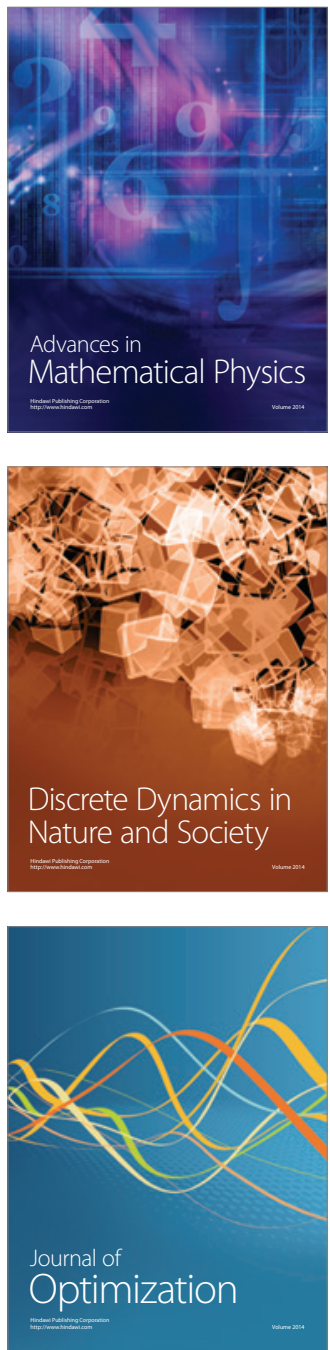